\title{
Characterization of the transcriptome profiles related to globin gene switching during in vitro erythroid maturation
}

\author{
Biaoru Li', Lianghao Ding ${ }^{2}$, Wei Li ${ }^{3}$, Michael D Story ${ }^{2}$ and Betty S Pace ${ }^{1 *}$
}

\begin{abstract}
Background: The fetal and adult globin genes in the human $\beta$-globin cluster on chromosome 11 are sequentially expressed to achieve normal hemoglobin switching during human development. The pharmacological induction of fetal $\gamma$-globin $(H B G)$ to replace abnormal adult sickle $\beta^{S}$-globin is a successful strategy to treat sickle cell disease; however the molecular mechanism of $\gamma$-gene silencing after birth is not fully understood. Therefore, we performed global gene expression profiling using primary erythroid progenitors grown from human peripheral blood mononuclear cells to characterize gene expression patterns during the $\gamma$-globin to $\beta$-globin $(\gamma / \beta)$ switch observed throughout in vitro erythroid differentiation.

Results: We confirmed erythroid maturation in our culture system using cell morphologic features defined by Giemsa staining and the $\gamma / \beta$-globin switch by reverse transcription-quantitative PCR (RT-qPCR) analysis. We observed maximal $\gamma$ globin expression at day 7 with a switch to a predominance of $\beta$-globin expression by day 28 and the $\gamma / \beta$-globin switch occurred around day 21. Expression patterns for transcription factors including GATA1, GATA2, KLF1 and NFE2 confirmed our system produced the expected pattern of expression based on the known function of these factors in globin gene regulation. Subsequent gene expression profiling was performed with RNA isolated from progenitors harvested at day 7, 14, 21, and 28 in culture. Three major gene profiles were generated by Principal Component Analysis (PCA). For profile-1 genes, where expression decreased from day 7 to day 28, we identified 2,102 genes downregulated $>1.5$-fold. Ingenuity pathway analysis (IPA) for profile-1 genes demonstrated involvement of the Cdc42, phospholipase C, NF-K $\beta$, Interleukin-4, and p38 mitogen activated protein kinase (MAPK) signaling pathways. Transcription factors known to be involved in $\gamma$-and $\beta$-globin regulation were identified. The same approach was used to generate profile-2 genes where expression was up-regulated over 28 days in culture. IPA for the 2,437 genes with > 1.5-fold induction identified the mitotic roles of polo-like kinase, aryl hydrocarbon receptor, cell cycle control, and ATM (Ataxia Telangiectasia Mutated Protein) signaling pathways; transcription factors identified included KLF1, GATA1 and NFE2 among others. Finally, profile-3 was generated from 1,579 genes with maximal expression at day 21 , around the time of the $\gamma / \beta$-globin switch. IPA identified associations with cell cycle control, ATM, and aryl hydrocarbon receptor signaling pathways.
\end{abstract}

Conclusions: The transcriptome analysis completed with erythroid progenitors grown in vitro identified groups of genes with distinct expression profiles, which function in metabolic pathways associated with cell survival, hematopoiesis, blood cells activation, and inflammatory responses. This study represents the first report of a transcriptome analysis in human primary erythroid progenitors to identify transcription factors involved in hemoglobin switching. Our results also demonstrate that the in vitro liquid culture system is an excellent model to define mechanisms of global gene expression and the DNA-binding protein and signaling pathways involved in globin gene regulation.

Keywords: Gene profiling, Erythroid maturation, $\gamma$-globin, $\beta$-globin, Hemoglobin switching, Fetal hemoglobin

\footnotetext{
* Correspondence: bpace@georgiahealth.edu

'Department Pediatrics, Georgia Health Sciences University, 1120 15th St.

CN-4112, Augusta, GA 30912, USA

Full list of author information is available at the end of the article
}

\section{Biomed Central}




\section{Background}

The fetal and adult globin genes in the $\beta$-globin cluster on chromosome 11 are expressed in a stage-specific manner during development to achieve the normal $\gamma / \beta$ globin gene switch after birth [1-4]. A large group of hemoglobin disorders result from mutations in the $\beta$ like globin genes including sickle cell anemia caused by an A to T mutation in $H B B$ ( $\beta$-globin) at the sixth position to produce $\beta^{\mathrm{S}}$-globin [5]. The association of two $\alpha$ globin chains with two $\beta^{\mathrm{S}}$-globin subunits forms hemoglobin $S$ which undergoes non-covalent polymerization due to abnormal intermolecular contacts under low oxygen conditions. This produces red blood cell sickling leading to the clinical symptoms observed in sickle cell anemia [6].

Extensive research has shown the beneficial effect of $\gamma$ globin reactivation by pharmacologic methods to induce fetal hemoglobin as a treatment modality for sickle cell patients. One such drug hydroxyurea was approved in 1998 [7]. Numerous laboratories have ongoing efforts to identify additional less toxic agents that induce fetal hemoglobin however few have reach clinical trials $[8,9]$. Therefore defining molecular mechanisms of globin gene regulation provides an approach to define specific strategies for $\gamma$-globin gene reactivation. With the availability of high throughput genomic methods, research aimed at the discovery of global mechanisms of gene regulation using in vitro models is now feasible [10] to establish personalized medical therapy [11].

To date, a limited number of transcriptome profiles have been reported for global genomic analysis in human erythroid cells. For example, K562 cells induced with hemin [12] were used to characterize transcriptomes related to drug induced erythroid differentiation. Subsequently, five studies have been published to characterize gene profiling during normal human erythroid differentiation using in vitro liquid culture systems [13-17]. Recently, Merryweather-Clarke et al. [17] used peripheral blood mononuclear cells combined with fluorescent activated cell sorting for the CD71 and CD36 surface markers. They analyzed expression data generated from erythroid progenitors isolate at the various stages of erythropoiesis and identified proteins with undiscovered function in erythroblast. Collectively, these studies generated significant findings regarding the erythroid transcriptome however our study is the first to characterize the transcriptome associated with the $\gamma / \beta$ globin switch.

To achieve this end, microarray analysis was performed using the Illumina whole genome platform to define global gene expression patterns associated with the $\gamma / \beta$-globin switch during primary erythroid maturation. We observed maximal $\gamma$-globin and $\beta$-globin gene expression at day 7 and 28 respectively with the $\gamma / \beta$-globin switch occurring around day 21 . We defined three major gene profiles consistent with a potential role of $\gamma$ globin activator (profile-1) with gene silencing from day 7 to day 28 ; $\beta$-globin activator (profile-2) showing increased gene transcription from day 7 to day 28 and profile- 3 genes defined by maximal expression at day 21 when the $\gamma / \beta$-globin switch was observed. Using $>1.5$ fold change in expression, we identified 2,102 profile- 1 genes some of which were involved in cell signaling through the p38 and ERK MAPK and erythropoietin receptor pathways. Another 2,437 profile-2 genes with patterns consistent with a positive role in $\beta$-globin regulation and 1,579 profile- 3 genes that might play a role in the $\gamma / \beta$-globin switch were identified. A global approach to define the transcriptome involved in globin gene expression during erythropoiesis can be used to generate testable hypotheses of $\gamma$-globin regulation and the development of strategies for fetal hemoglobin induction to treat sickle cell anemia.

\section{Results and discussions}

The $\gamma / \beta$-globin switch is observed in the one-phase liquid culture system

To study global mechanisms of globin gene regulation, we established the in vitro one-phase liquid culture system developed by Uddin et al. [18]. We modified this system using, human peripheral blood mononuclear cells grown in the presence of stem cell factor $(50 \mathrm{ng} /$ $\mathrm{mL})$, Interleukin-3 (50 ng/mL), and erythropoietin $4 \mathrm{IU} /$ $\mathrm{mL}$ from day 0 . Cells were harvested every two to three days for cell morphology by Giemsa stain and $\gamma$-globin and $\beta$-globin gene mRNA levels were quantified by RTqPCR analysis (see Methods). From day 16 to day 31, the percentage of early and late erythroid progenitors increased from $5 \%$ to $84 \%$ (Figure 1A). Early erythroid cells possess a deep blue cytoplasm consistent with basophilic erythroblasts followed by maturation into late orthochromatophilic erythroblasts by day 28 (data not shown). During the same time period $\gamma$-globin and $\beta$ globin gene expression was monitored by RT-qPCR. We observed progressive $\gamma$-globin silencing and concomitant $\beta$-globin activation with the $\gamma / \beta$-globin switch occurring around day 21 (Figure 1B) recapitulating human hemoglobin switching observed during development.

We next analyzed the response of the $\gamma$-globin gene in more detail to establish our system as a good model for performing transcriptome analysis. At birth, fetal hemoglobin composes $80-90 \%$ of the total hemoglobin synthesized and it gradually decreases to $<1 \%$ by 10 months in normal infants [19]. Fetal hemoglobin is a heterogeneous mixture of $\gamma$-globin polypeptide chains containing either glycine $(\mathrm{G} \gamma)$ or alanine $(\mathrm{A} \gamma)$ at residue 
A

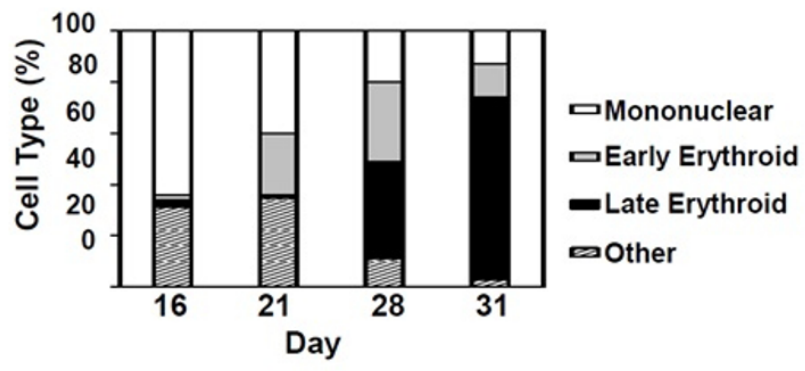

B

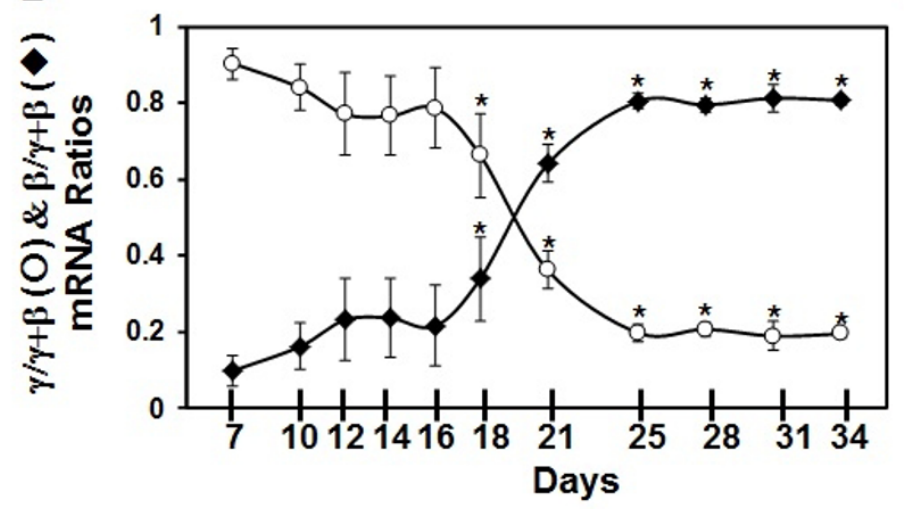

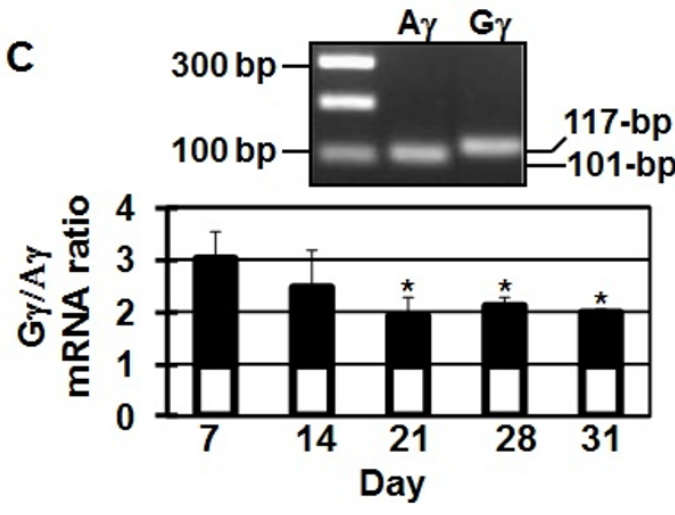

D

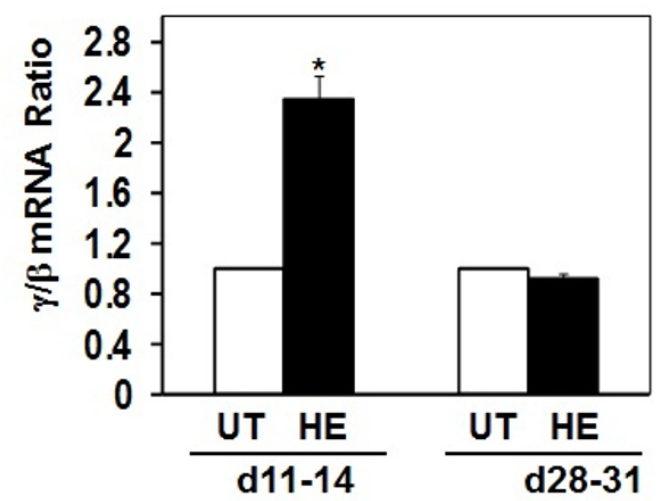

Fig. 1 A-D

Figure 1 The $\gamma / \beta$-globin switch is recapitulated in the one-phase liquid culture system. Cells grown in the one-phase system were harvested every 2-3 days and analyzed as follows (see Methods). A) Cell morphology was determined by Giemsa stain and different cell types classified based on standard morphologic features. B) RT-qPCR analysis was performed to determine changes in $\gamma$-globin and $\beta$-globin mRNA

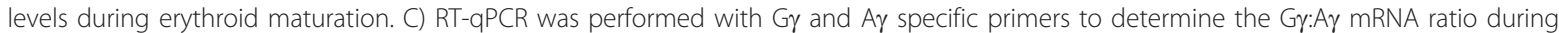
erythropoiesis. A $\gamma$-globin expression was normalized to one (white bars) and the relative changes in G $\gamma$-globin mRNA are shown in the black bars. D) Progenitors were induced on day 11 or day 28 with $50 \mu \mathrm{M}$ hemin (HE) for 72 hrs and then RT-qPCR was completed.

136 [20]. At birth G $\gamma$-chains predominate, however, a switch to predominantly A $\gamma$-chains arises during the first year of life going from a 3:1 to $1: 1 \mathrm{G} \gamma: \mathrm{A} \gamma$ ratio. As shown in Figure $1 C$, the ratio of $\mathrm{G} \gamma: \mathrm{A} \gamma$-globin expression changed from $2: 1$ at day 7 , to $1: 1$ by day 31 a pattern similar to that observed after birth [21].

Subsequently, we tested the ability to activate $\gamma$-globin expression in our human erythroid culture system using hemin, a known fetal hemoglobin inducer. Hemin activated $\gamma$-globin 2.3 -fold at day 11 in contrast to a lack of induction at day 28 (Figure 1D) suggesting the ability of hemin to further enhance a transciptionally active $\gamma$-globin gene at day 11 . By contrast, day 28 cultured cells carrying a silenced $\gamma$-globin gene were resistant to induction by hemin. These data established our onephase liquid culture as a model to study erythropoiesis that can be used to define the erythroid transcriptome associated with the $\gamma / \beta$-globin switch.

\section{Data mining approach}

Based on the timing of the $\gamma / \beta$-globin gene switch, we performed microarray analysis to define the erythroid cells transcriptome. Our data mining approach (Figure 2) consisted of four steps: 1) data normalization after microarray chip hybridization using Model-Based Background Correction (MBCB) for BeadArrays and quartile normalization; 2) time-course gene expression profiling using PCA and the confirmation of gene profiles for a subset of genes by RT-qPCR analysis; 3) gene classification using DAVID (the Database for Annotation, Visualization and Integration Discovery) gene ontogeny (GO); and 4) functional genomics to define the signaling 


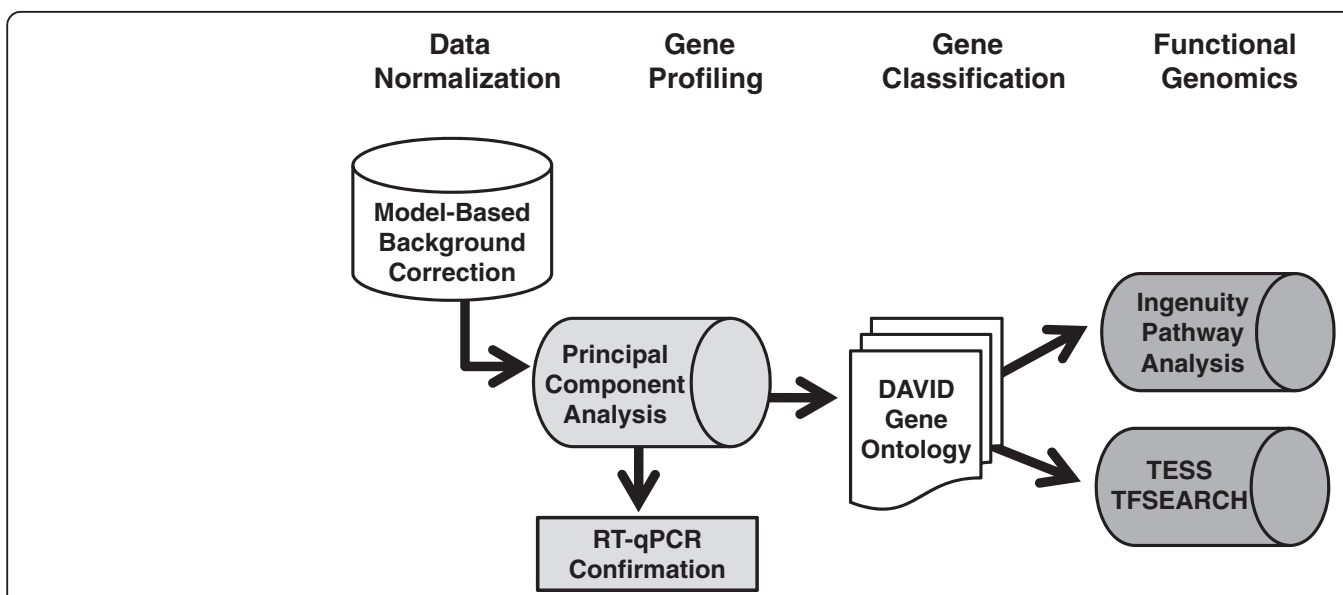

Figure 2 Data mining strategy. Shown is a schematic diagram outlining our genomic bioinformatics approach for the data generated by microarray analysis.

pathways potentially involved in globin regulation using Ingenuity Pathway Analysis (IPA). In silico analysis with Transcription Element Search Site (TESS), TFSEARCH, Weeder $\mathrm{H}$, and FIRE platforms was also performed to identify transcription factors with predicted binding sites in the $\beta$-globin locus.

\section{Data collection and gene expression profiling}

We collected three RNA samples at day 7, 14, 21, and 28 which allowed us to perform gene expression profiling over a time course. The advantage of this approach is the use of well-established time-course algorithms for data mining. However, the variability that can occur at different times in culture can introduce errors in gene expression data, but replicate samples help to address this concern. RNA quality check was performed prior to microarray analysis on the Illumina HumanWG-6V2 Expression BeadChip containing 48,700 probes on the Bio-Rad Experion system. Automated electrophoresis qualities confirmed RQI (RNA Quality Index) values > 8.0 for all samples included in our microarray analysis (Additional file 1: Figure S1). The raw data generated are summarized in Additional file 2: Table S1, Additional file 3: Table S2, Additional file 4: Table S3, Additional file 5: Table S4.

After the raw data was normalized several gene probes including $S 18, S 28, D D X 5$ and $A C T B$ were used to determine data consistency. The results show no significant differences $(p>0.05)$ in expression across all microarrays for the internal control genes. We also observed that the expression of erythroid cell biomarkers such as CD36 and glycophorin A (GPA) significantly increased (44-fold to 266-fold) from day 7 to day $28(p<0.001)$ (Figure 3A); however CD71 levels increased 7 -fold $(p<0.001)$ followed by slightly decreased expression by day 28. These findings are consistent with the expression patterns of cell surface markers during normal human erythroid differentiation [22,23].

We next examined the expression profiles of genes known to be involved in $\gamma$-globin and $\beta$-globin regulation prior to global data mining. The following genes including GATA2 [24], cJun [25], KLF2 [26], SATB1 [27], KLF4 [28], FOS [29], IKZF1 [30] and RUNX2 [31], which have been reported to be associated with $\gamma$-globin regulation were analyzed (Figure 3B). Simultaneous with $\gamma$-globin silencing, we observed decreased expression from day 7 to day 28 for GATA-1,KLF2, KLF4, FOS, SABT1, cJun, IKFZ1 and RUNX2 among others in agreement with published data.

Genes previously shown to be associated with $\beta$-globin regulation including KLF1 [32], GATA1 [33], NFE2 [34], FOG1 [35] and FOXM1 [16] were examined as well. We observed significant KLF1 (108-fold; $p<0.01$ ) and GATA1 (112-fold) induction compared to 17.5-fold induction of NFE2, FOG1 (4.6-fold) and FOXM1 (9.6fold) from day 7 to day 28, findings consistent with published results (Figure $3 \mathrm{C}$ ). These data support our line of reasoning that whole genome gene profiling might identify other transcription factors involved in globin gene regulation.

Before data mining, the normalized microarray data was examined in a multiple regression algorithm to study chip-chip reproducibility (Additional file 1: Figure $\mathrm{S} 4 \mathrm{~A})$. For the three chips analyzed at day 7 the adjusted $\mathrm{R}^{2}=0.99$ (F-value 4.94E + 0.6 and P-value 2.2E-16), for day 14 triplicates the adjusted $R^{2}=0.98$ (F-value $2.96 \mathrm{E}$ +0.6 and P-value 2.2E-16), for day 21 chips, the adjusted $R^{2}=0.99(F$-value $3.51 E+0.6$ and $P$-value $2.2 \mathrm{E}-16)$, and for day 28 triplicates the adjusted $\mathrm{R}^{2}=$ 

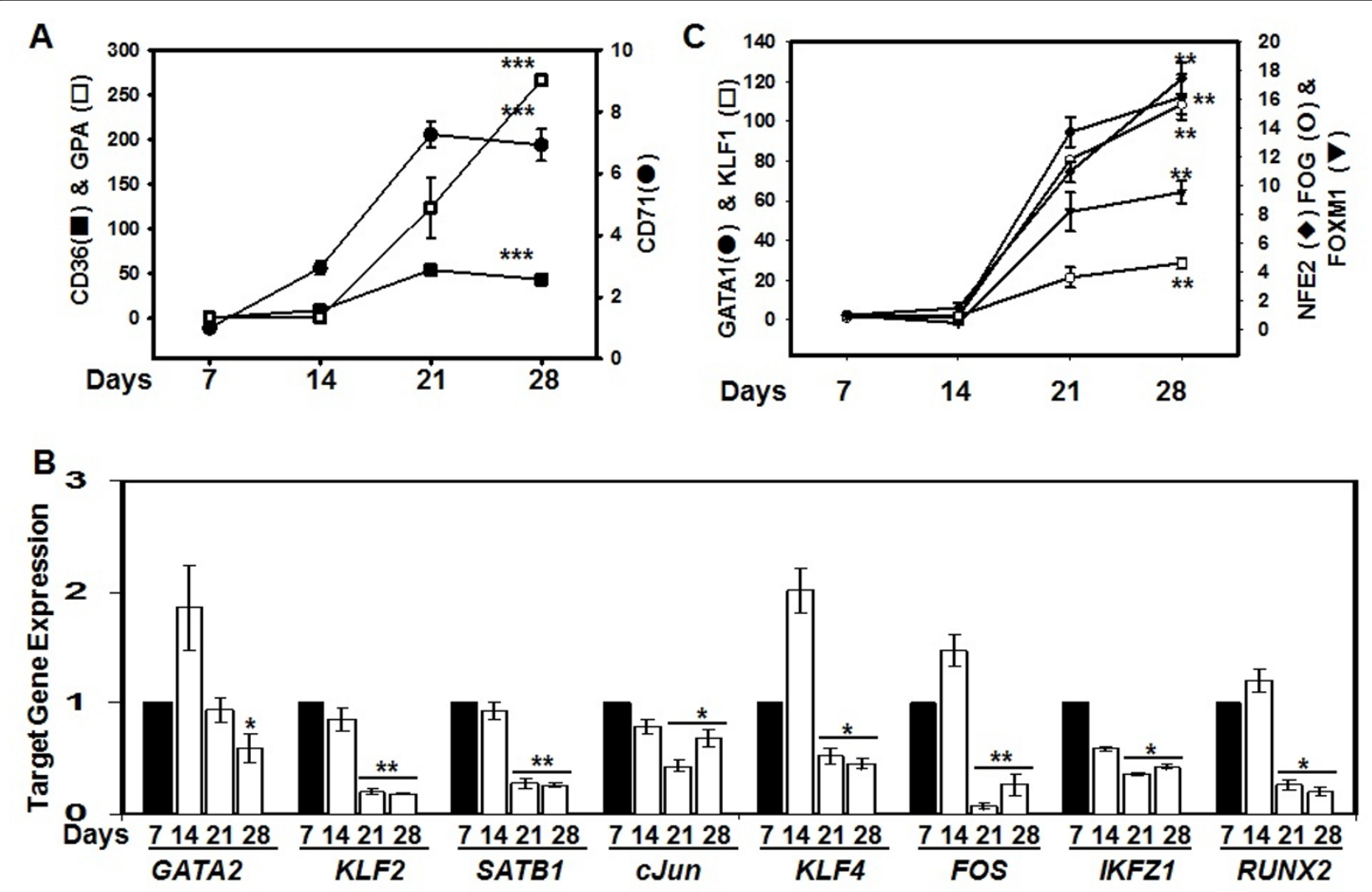

Fig. 3 A-C

Figure 3 Gene expression analysis of transcription factors known to regulate globin gene expression. A) Gene expression patterns for established erythroid markers are shown. Note the different scales for the left and right Y-axis to reflect the magnitude of difference in gene expression. Shown is the mean $\pm \mathrm{SEM} ;{ }^{* * *}=p<0.001$. B) Time-course curves of genes previously reported to be involved in $\gamma$-globin regulation are shown; ${ }^{*}=p<0.05 ;{ }^{*}=p<0.01$. C) Graphed is the expression profiles for genes known to regulate $\beta$-globin expression. The raw data for the genes shown are included in Additional file 2: Table S1, Additional file 3: Table S2, Additional file 4: Table S3, Additional file 5: Table S4.

0.99 (F-value 4.92E + 0.6 and P-value 2.2E-16). These data demonstrate a high correlation of data reproducibility between microarray chips.

Initial gene profiling studies were conducted by Principal Component Analysis (PCA), which is an approach to identify patterns in large datasets. PCA involves a multivariate mathematical procedure that transforms a number of correlated variables into a smaller number of uncorrelated variables called principal components. The first principal component accounts for as much of the variability in the dataset as possible, and each succeeding component accounts for the remaining variability. Normalized data were used for PCA to define three major expression profiles including genes silenced from day 7 to day 28 (profile-1), genes activated over the culture period (profile-2) and genes with maximal expression at day 21 (profile-3) during the time of the $\gamma / \beta$ globin switch.
Because our genomics data were mined to follow the expression patterns of the $\gamma$ - and $\beta$-globin genes patterns, we chose a statistical analysis consisting of F-distribution with the Analysis of Variance (ANOVA) value $=0.01$ and False Discovery Rate $(\mathrm{FDR})=0.05$. Our goal was to maintain globin gene expression profiles therefore permutation analysis was not conducted. To establish gene subsets for our subsequent functional genomics analysis, we identified gene with $>1.25-$, > 1.5 - and $>2$-fold change in expression from day 7 to day 28 by PCA (Table 1). For the highest stringency of 2-fold, we observed 492, 357, and 325 genes for profile$1,-2$ and -3 respectively. The PCA data for genes with > 1.5-fold differential expression are summarized in Additional file 6: Table S5; these gene were used for the hierarchical cluster studies shown in Figure 4B which further illustrates the three major gene profiles consistent with the PCA data. 
Table 1 Summary of Principle Component Analysis

\begin{tabular}{lccc}
\hline & $>$ 1.25-fold & $>$ 1.5-fold* & $>$ 2-fold \\
\hline profile-1 & 3,317 & 1,610 & 492 \\
\hline profile-2 & 3,105 & 2,080 & 357 \\
\hline profile-3 & 2,120 & 1,254 & 325 \\
\hline
\end{tabular}

*Genes with $>1.5$-fold change were used for the subsequent bioinformatics analyses

It is established practice to confirm the expression pattern obtained by microarray with RT-qPCR analysis. Therefore, we confirmed the microarray data for 37 experimental genes chosen from profile-1, profile-2, and internal controls. As shown in Table 2, 93.5\% (29 out of 31) of gene expression patterns identified by microarray analysis were confirmed by RT-qPCR. Of note are similar patterns for the hematopoietic transcription factors KLF1, NFE2 and GATA1 however the magnitude of change was different. To further validate our microarray data, regression analysis was completed for the two data sets. Our results showed that gene expression patterns obtained by RT-qPCR analysis correlated to that observed by microarray assay. For example, profile- 1 genes including GATA2 showed $\mathrm{R}^{2}=0.80, p=0.104$ and for $S A T B 1 \mathrm{R}^{2}=0.977, p=0.012$. The same analysis for profile- 2 genes showed $K L F 1$ with a $\mathrm{R}^{2}=0.91, p=$ 0.045, GATA1 $\left(\mathrm{R}^{2}=0.99, p=0.003\right)$, NFE2 $\left(\mathrm{R}^{2}=0.755\right.$, $p=0.131)$ and FOG1 $\left(\mathrm{R}^{2}=1, p=0.00\right)$. However, not all fold change in gene expression correlate between the two techniques which have been reported by other investigators [36,37]. In our study, $c-M y c$, and $18 S$ show disparate results that might reflect differences in sensitivity of the two techniques. Inconsistencies for $18 \mathrm{~S}$ rRNA expression due to technical issue such as mRNA degradation have been reported [38]. Overall, we observed a high correlation between data generated by microarray and RT-qPCR to support the accuracy of our gene profiling approach.

\section{DAVID gene ontogeny (GO) gene function analysis}

To properly classify gene function, the transcriptome generated from microarray analysis was analyzed by GO software to: a) address whether or not the data mining correctly identified related genes expressed in the expected cell type using the Kappa score; b) define functional annotation and transcriptome categories based on biological processes, cellular components and molecular function to address the enriched relationships among many genes; and c) reflect the individual GO functional level by over-representation with higher numbers of genes and significant p-values in the GO terms to determine and classify GO groups $[39,40]$. We completed a GO clustering analysis for each gene profile subset with $>1.5$-fold change in expression during erythroid maturation using the DAVID platform [41]. For interpretation of our analysis, the higher the Kappa score for a given genomic profile, the stronger the agreement with the cell type from which the specimens were extracted; if Kappa $=1$, then there is perfect agreement

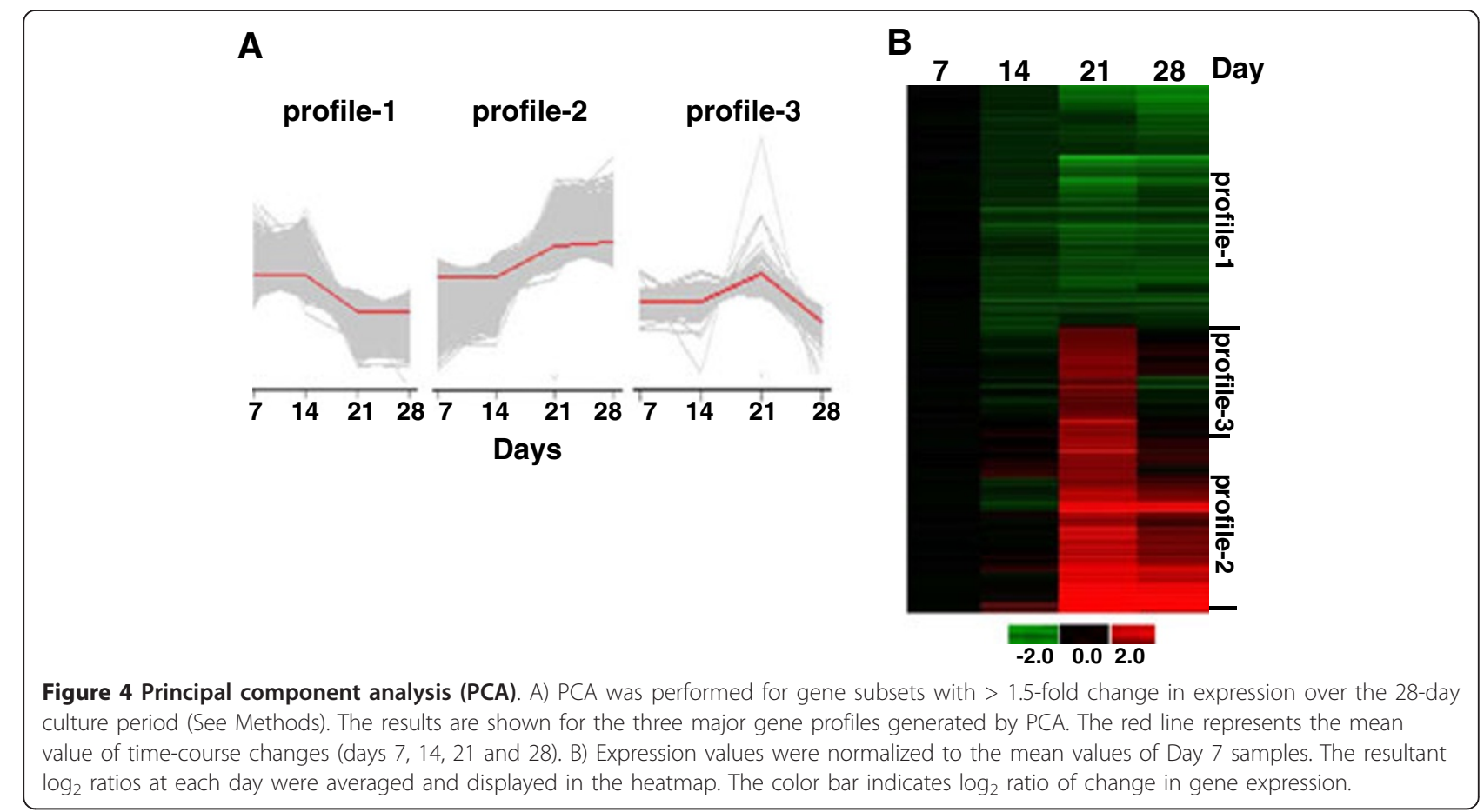

B 
Table 2 Comparison between microarray and RT-qPCR mRNA levels

\begin{tabular}{|c|c|c|c|c|c|c|c|c|c|c|}
\hline \multirow[t]{2}{*}{ Number } & \multirow[t]{2}{*}{ Gene sources } & \multirow[t]{2}{*}{ Symbol } & \multicolumn{4}{|c|}{ Microarray(Mean fold change) } & \multicolumn{4}{|c|}{ RT-qPCR(Mean fold change) } \\
\hline & & & D7 & D14 & D21 & D28 & D7 & D14 & D21 & D28 \\
\hline 1 & profile-1 & STAT5A & 1.00 & 0.50 & 0.99 & 0.35 & 1.00 & 1.18 & 0.56 & 0.46 \\
\hline 2 & profile-1 & $D B P$ & 1.00 & 1.13 & 0.28 & 0.67 & 1.00 & 0.08 & 0.28 & 0.48 \\
\hline 3 & profile-1 & FOXQ1 & 1.00 & 0.66 & 0.57 & 0.54 & 1.00 & 0.00 & 0.05 & 0.03 \\
\hline 4 & profile-1 & NFIL3 & 1.00 & 0.75 & 0.23 & 0.19 & 1.00 & 0.10 & 0.35 & 0.26 \\
\hline 5 & profile-1 & MTF1 & 1.00 & 0.66 & 0.50 & 0.75 & 1.00 & 0.58 & 0.97 & 0.82 \\
\hline 6 & profile-1 & JUN & 1.00 & 0.79 & 0.43 & 0.68 & 1.00 & 0.05 & 1.10 & 0.22 \\
\hline 7 & profile-1 & KLF2 & 1.00 & 0.86 & 0.20 & 0.18 & 1.00 & 0.08 & 0.11 & 0.11 \\
\hline 8 & profile-1 & CEBPA & 1.00 & 1.20 & 0.09 & 0.10 & 1.00 & 0.02 & 0.18 & 0.17 \\
\hline 9 & profile-1 & CREBBP & 1.00 & 0.85 & 0.70 & 0.99 & 1.00 & 0.81 & 0.65 & 0.61 \\
\hline 10 & profile-1 & SP3 & 1.00 & 0.84 & 0.88 & 0.87 & 1.00 & 0.98 & 0.85 & 0.47 \\
\hline 11 & profile-1 & SATB1 & 1.00 & 0.94 & 0.28 & 0.26 & 1.00 & 0.80 & 0.24 & 0.15 \\
\hline 12 & profile-1 & $c-M y c$ & 1.00 & 0.95 & 1.05 & 0.62 & 1.00 & 11.9 & 14.63 & 11.95 \\
\hline 13 & profile-1 & GATA2 & 1.00 & 1.87 & 0.95 & 0.59 & 1.00 & 4.00 & 1.20 & 1.20 \\
\hline 14 & profile-1 & KLF11 & 1.00 & 1.22 & 0.58 & 0.70 & 1.00 & 2.16 & 0.58 & 0.70 \\
\hline 15 & profile-2 & FOG1 & 1.00 & 0.96 & 3.64 & 4.63 & 1.00 & 0.99 & 3.70 & 4.70 \\
\hline 16 & profile-2 & USF1 & 1.00 & 1.01 & 0.85 & 1.26 & 1.00 & 0.71 & 1.45 & 2.02 \\
\hline 17 & profile-2 & SP1 & 1.00 & 0.86 & 1.20 & 1.55 & 1.00 & 1.16 & 1.02 & 1.39 \\
\hline 18 & profile-2 & KLF3 & 1.00 & 0.95 & 1.45 & 1.86 & 1.00 & 0.95 & 1.83 & 1.72 \\
\hline 19 & profile-2 & KLF1 & 1.00 & 1.75 & 80.72 & 108.50 & 1.00 & 2.22 & 6.70 & 13.80 \\
\hline 20 & profile-2 & POU2F1 & 1.00 & 0.91 & 1.28 & 1.90 & 1.00 & 0.57 & 1.23 & 1.16 \\
\hline 21 & profile-2 & KLF13 & 1.00 & 0.98 & 1.12 & 1.83 & 1.00 & 2.16 & 0.58 & 8.70 \\
\hline 22 & profile-2 & GATA1 & 1.00 & 1.83 & 94.63 & 112.31 & 1.00 & 1.80 & 7.40 & 8.40 \\
\hline 23 & profile-2 & $N F-E 2$ & 1.00 & 1.53 & 10.98 & 17.47 & 1.00 & 17.6 & 85.80 & 776.40 \\
\hline 24 & profile-2 & ATF2 & 1.00 & 1.25 & 1.69 & 2.03 & 1.00 & 2.70 & 1.50 & 2.40 \\
\hline 25 & profile-2 & FGFR3 & 1.00 & 1.20 & 47.87 & 39.77 & 1.00 & 8.22 & 956. & 923.45 \\
\hline 26 & profile-2 & MAPKK6 & 1.00 & 1.01 & 1.65 & 2.07 & 1.00 & 1.39 & 1.13 & 2.12 \\
\hline 27 & profile-2 & HSP9OkDa & 1.00 & 0.95 & 1.65 & 1.75 & 1.00 & 0.63 & 1.50 & 8.57 \\
\hline 28 & profile-2 & v-Myc & 1.00 & 1.03 & 1.70 & 2.49 & 1.00 & 30.5 & 0.45 & 2.44 \\
\hline 29 & profile-2 & CUTL1 & 1.00 & 1.02 & 1.58 & 2.20 & 1.00 & 1.02 & 0.42 & 2.06 \\
\hline 30 & Reference 1 & $A C T B$ & 1.00 & 1.05 & 0.84 & 0.91 & 1.00 & 1.00 & 1.00 & 1.00 \\
\hline 31 & Reference 2 & $18 \mathrm{~S}$ & 1.00 & 0.96 & 1.00 & 1.06 & 1.00 & 0.68 & 1.62 & 1.41 \\
\hline
\end{tabular}

for cell type. The detailed results and corresponding $\mathrm{p}$ values for each GO term are shown in Additional file 7: Table S6.

For profile- 1 genes, Kappa $=1$ was obtained for the GO term hematopoiesis (Enrichment score 15.91, $p=$ 7.7E-10). Profile 2 genes were highly associated with the GO term erythrocyte differentiation, Kappa $=1$ (Enrichment score 2.5, $p=1.5 \mathrm{E}-4$ ). On the other hand, profile3 genes had a Kappa = 1 for the GO term macromolecular complex subunit organization (Enrichment score 5.27, $p=3.8 \mathrm{E}-7)$. This latter term describes a process by which macromolecules aggregate, or disaggregate to reform, disassembly, or alter macromolecular complexes. Reorganization of these complexes has been largely reported in protein expression changes and gene switching produced in cells infected with viruses, bacteria and parasite [42]. We speculate that macromolecule complex reorganization may occur during the $\gamma / \beta$-globin switch.
To further characterize the genes identified in each profile we used over-representation to classify GO groups. In this analysis we investigated two major GO categories where 1) biological processes with 30 subgroups and 2) molecular function with 20 subgroups were identified $(p<0.05)$. For biological processes, the following subgroups are highlighted for profile-1: 47 genes were over-represented in hemopoiesis (Enrichment score 15.91, $p=7.7 \mathrm{E}-10)$ and 85 genes in cell activity (Enrichment score 15.91, $p=1.4 \mathrm{E}-29$ ). We also observed 121 profile-2 genes related to cellular proliferation (Enrichment score 25.4, $p=1.43 \mathrm{E}-32$ ) and 8 genes related to heme biosynthesis (Enrichment score 3.27, $p$ $=6.5 \mathrm{E}-6)$. Finally, 81 genes in profile- 3 were associated with nucleotide metabolism and DNA processing (Enrichment score 5.14, $p=1.1 \mathrm{E}-6$ ). The GO subgroups identified would be predicted since erythropoiesis involves actively dividing hematopoietic cells, which 
require heme biosynthesis for normal hemoglobin production.

For the second GO category molecular function, profile-1 genes were over-represented in the hydrolase category including protein tyrosine phosphatase, MAP kinase phosphatase, GTP cyclohydrolase and tyrosine/ serine/threonine phosphatase. By contrast, molecular function GO terms for profile-2 genes included iron, heme and oxygen binding proteins; profile- 3 genes were related to adenyl nucleotide, ATP and nucleoside binding proteins. In summary, DAVID GO data mining classified profile-1 genes as associated with hematopoiesis while profile- 2 genes were related to cell proliferation and erythrocyte differentiation. Finally, profile- 3 genes were associated with alteration of a macromolecular complex, or protein switching processes requiring DNA synthesis.

\section{Functional genomics - Ingenuity Pathway Analysis (IPA)}

IPA is software that helps researchers model, analyze, and understand complex systems by integrating data from a variety of experimental platforms and providing insight into molecular and chemical interactions, cellular phenotypes, and disease processes. We performed IPA to identify pathways involved in erythropoiesis defined at $p<0.05$ and IPA significance value $>1.3$.

The IPA for profile- 1 genes with $>1.5$-fold decreased expression over 28 days is shown in Table 3 . The most highly correlated pathways included Cdc42 (cell division cycle 42, GTP binding protein) (36 hits, $p=14.2$ ), NFK $\beta$ (33 hits, $p=7.92$ ), Phospholipase $C$ (42 hit, $p=$ 7.13), Interleukin 4 (17 hits, $p=4.78)$, LXR/RXR (15 hits, $p=3.63)$ and erythropoietin (14 hits, $p=3.17$ ).

Table 3 Main pathways identified by profile- 1 genes

\begin{tabular}{ccc}
\hline $\begin{array}{c}\text { Ingenuity Canonical } \\
\text { Pathways }\end{array}$ & gene hits & -log (p-value) \\
\hline Cdc42 & 36 & 14.2 \\
Phospholipase C & 42 & 7.13 \\
NF- $\kappa \beta$ & 33 & 7.92 \\
Interleukin-4 & 17 & 4.78 \\
LXR/RXR Activation & 15 & 3.63 \\
Erythropoietin & 14 & 3.17 \\
PPAR $/$ RXR $\perp \pm$ Activation & 21 & 2.19 \\
Rac & 16 & 2.17 \\
SAPK/NK & 14 & 1.92 \\
P7OS6K & 17 & 1.88 \\
Acute Phase Response & 21 & 1.66 \\
Notch & 7 & 1.64 \\
PPAR & 12 & 1.56 \\
ERK/MAPK & 21 & 1.44 \\
P38 MAPK & 13 & 1.44 \\
Insulin Receptor & 16 & 1.43 \\
\hline
\end{tabular}

Interestingly, the $C d c 42$ gene is not differentially expressed by microarray and RT-qPCR data (Additional file 2: Table S1, Additional file 3: Table S2, Additional file 4: Table S3, Additional file 5: Table S4 and Table 2 respectively). Although mRNA levels may not be altered, Cdc42 signaling can be activated by phosphorylation at serine 71 [43] with subsequent activation of downstream effector molecules. The latter proteins such as $P A K 2$ or SPEC1 may be regulated at the transcriptional and posttranslational levels (Figure 5A) to explain the high score obtained for the Cdc42 pathway.

Although most of these pathways have not been directly associated with mechanisms of globin gene regulation, there are multiple studies to support the involvement of p38 MAPK signaling in erythropoiesis (Figure $5 \mathrm{~B})$. For instance by IPA, p38 MAPK is activated by phospholipase C [44], Cdc42 [45], and LXR/RXR [46] signaling. Although initially identified as a protein kinase activated by stress, p38 MAPK signaling coordinates cellular responses during erythropoiesis [47]. For example, p38 MAPK is essential for the synthesis of hemoglobin [48] and $\mathrm{p} 38 \alpha^{-/-}$mice exhibit severe anemia and die in utero owing to defects in angiogenesis, and placental insufficiency [49]. Furthermore, the proliferation and differentiation of erythroid progenitors is controlled by erythropoietin through the p38 MAPK pathway [50] and p38 is required for erythropoietin mRNA stability [51].

Previous data from our laboratory demonstrated that p38 MAPK signaling plays an important role in drugmediated fetal hemoglobin induction in primary erythroid cells [52]. Moreover, ATF2, CREBBP and cJun are required to induce $\gamma$-globin expression via p38 activation. The global pathway analysis from profile- 1 genes suggests that p38 MAPK plays a direct role in $\gamma$-globin gene regulation independent of drug induction. These data are in agreement with our recent publication demonstrating that $\mathrm{p} 38$ is required for steady-state $\gamma$ globin activation during normal erythropoiesis [53]. The specific role of other signaling pathways in globin gene regulation remains to be defined.

In contrast to efforts aimed at understanding mechanisms of $\gamma$-globin gene expression, fewer studies have focused on $\beta$-globin regulation via cell signaling pathways. In our pathway analysis of profile- 2 genes, we identified the Mitotic Roles of Polo-Like Kinase (PLK) pathway (19 hits, $p=7.22$ ) highly associated with $\beta$-globin activation (Table 4 and Additional file 1: Figure $\mathrm{S} 5 \mathrm{C})$. Although there is no direct evidence for PLK involvement in globin gene regulation, it is known that PLK can inhibit histone deacetylase 3 activity [54]. Furthermore, histone deacetylase 3 can inhibit the TATA box and produce positive regulation of $\beta$-globin expression by enhanced USF and TFII binding [55]. On the contrary, Perrine and colleagues demonstrated $\gamma$ - 


\section{A}

\section{Cdc42 pathway}

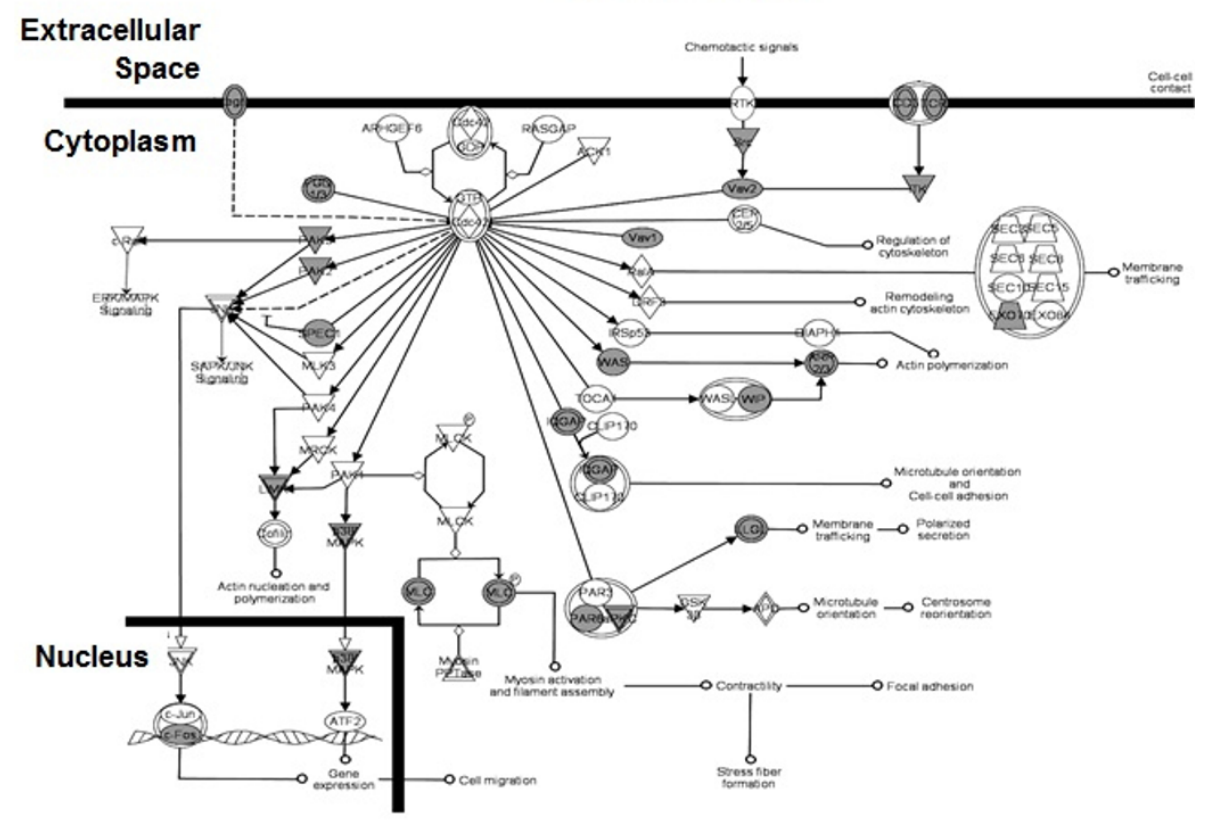

B

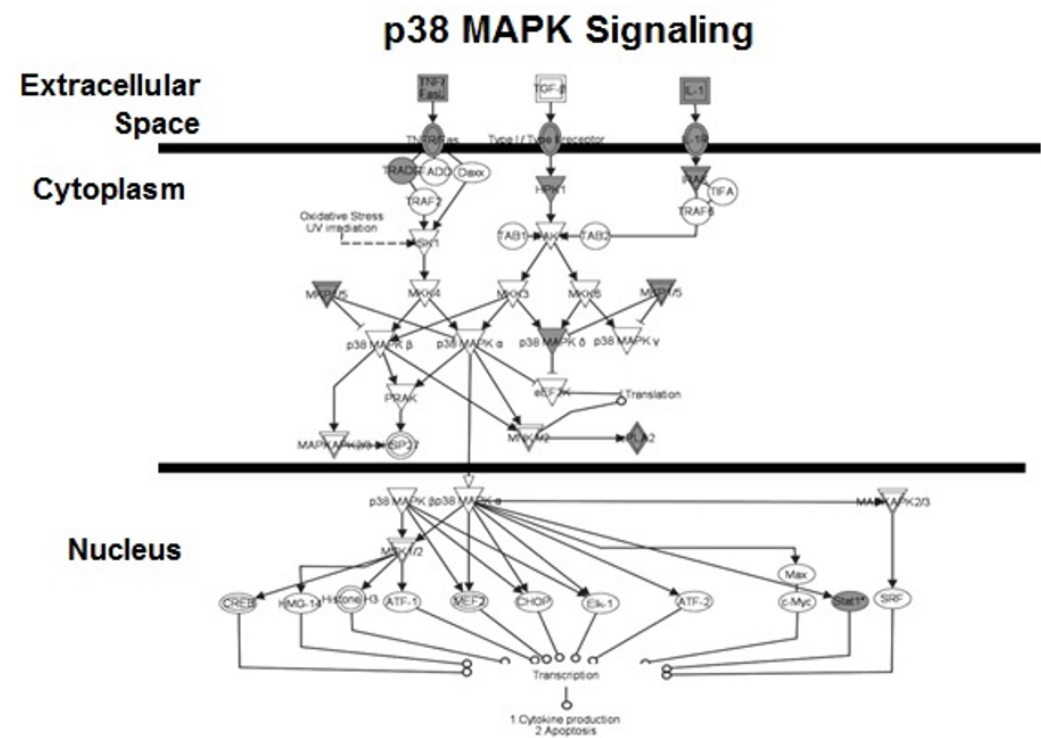

Figure 5 Pathways identified by IPA. Profile-1 genes were used for IPA. The A) Cdc42 and B) p38 MAPK signaling pathways identified for profile-1 genes are shown. The shaded symbols represent gene hits in the pathway and clear symbols representing other known genes in the signaling pathway (squares $=$ cytokines, oval shape $=$ transcription factors and triangle shape $=$ kinases).

globin gene silencing mediated by a HDAC3-NCoR repressor complex [56]. These finding suggest some protein may play a dual role in globin gene regulation.

Numerous other signaling pathways including the Aryl hydrocarbon receptor, ATM, cell cycle regulation, P53 protein and RAC are activated during adult stage $\beta$-globin gene expression $[57,58]$. Our studies for profile- 2 genes (Table 4) revealed the cell cycle control proteins, and Aryl hydrocarbon receptor, and ATM pathways with the highest number of gene hits. Of interest is the ATM pathway (Additional file 1: Figure S5D) which phosphorylates several proteins to activate the DNA damage checkpoint, leading to cell cycle arrest, apoptosis and cell differentiation [59]. This pathway also 
Table 4 Main pathways identified by profile- 2 genes

\begin{tabular}{lcc}
\hline Ingenuity Canonical Pathways & $\begin{array}{c}\text { gene } \\
\text { hits }\end{array}$ & $\begin{array}{c}-\mathbf{l o g}(\mathbf{p}- \\
\text { value) }\end{array}$ \\
\hline Mitotic Roles of Polo-Like Kinase & 19 & 7.22 \\
CHK Proteins in Cell Cycle Checkpoint Control & 14 & 5.96 \\
Cell Cycle: G2/M DNA Damage Checkpoint & 13 & 5.02 \\
Regulation & & \\
Aryl Hydrocarbon Receptor & 24 & 3.74 \\
ATM & 13 & 3.66 \\
Cell Cycle: G1/S Checkpoint Regulation & 11 & 2.34 \\
p53 & 15 & 2.04 \\
RAC & 16 & 1.90 \\
Cell Cycle Regulation by BTG Family Proteins & 7 & 1.62 \\
\hline
\end{tabular}

regulates DNAPK which has been associated with bone marrow failure [60]. Recent studies support a role for ATM in the regulation of erythropoiesis and $\beta$-globin expression however the exact mechanism remains unclear [61]. ATM was also identified with profile-3 genes (Table 5) suggesting this signaling pathway may play a more general role in erythroid maturation.

Published data to define the erythroid transcriptome in human primary progenitors [13-17] have mainly been established using a variety of liquid culture systems and a range of $0-43 \%$ differentially expressed genes have been reported. Given the differences in experimental design for the various studies it is difficult to directly compare results. The most recent study by Merryweather-Clarke et al. [17] is most similar with our design except they used the two-phase liquid culture design established by Fibach et al. [62] and a FACS sorting step to isolate purified erythroid progenitors for gene profiling. They reported the highest level of differentially expressed genes at $47 \%$ and identified potential new targets involved in erythroid maturation. By comparison, we identified 38\% differentially expressed genes and identified similar cellular pathways involved in erythroid maturation by IPA and DAVID GO analysis. Our

Table 5 Main pathways identified by profile- 3 genes

\begin{tabular}{lcc}
\hline Ingenuity Canonical Pathways & $\begin{array}{c}\text { gene } \\
\text { hits }\end{array}$ & $\begin{array}{c}- \text { log(p- } \\
\text { value) }\end{array}$ \\
\hline Role of CHK Proteins in Cell Cycle Checkpoint & 10 & 4.98 \\
Control & 10 & 3.17 \\
ATM & 9 & 2.40 \\
Cell Cycle: G1/S Checkpoint Regulation & 7 & 2.15 \\
Cell Cycle: G2/M DNA Damage Checkpoint & & \\
Regulation & 15 & 2.02 \\
Aryl Hydrocarbon Receptor Signaling & 4 & 1.91 \\
RAN & 19 & 1.86 \\
LPS/IL-1 Mediated Inhibition of RXR Function & 6 & 1.81 \\
Nucleotide Excision Repair Pathway & 9 & 1.34 \\
Fructose and Mannose Metabolism &
\end{tabular}

study is unique in that none of the published studies performed in silico transcription factor binding analysis based on major gene profiles to gain insights into the $\gamma /$ $\beta$ globin switching described below.

\section{Functional genomics - identification of $\beta$-locus transcription factor binding sites}

In the final set of analyses our goal was to identify transcription factor binding sites for profile-1, -2 , and -3 proteins identified by PCA. The regions shown in Figure $6 \mathrm{~A}$ and $6 \mathrm{~B}$ were chosen based on the speculation that profile-1 genes (silenced from day 7 to day 28) might be trans-activators of $\gamma$-globin expression since they are expressed in a similar pattern as other factors demonstrated to activate $\gamma$-globin. Likewise, profile- 2 genes (activated from day 7 to day 28) might be involved in $\beta$ globin regulation. The alternative possibility that profile1 and profile- 2 genes are $\beta$ - or $\gamma$-globin silencers respectively was not tested. With this goal in mind, we performed in silico analysis using TESS, TFSEARCH, Weeder $\mathrm{H}$, and FIRE platforms and DNA sequences from the Human Genome (Hg19 version; NG_00007.3) to identify predicted binding sites in the $\beta$-locus. Shown in Figure 6 are schematics of the regions targeted for these analyses. We examined the locus control region (LCR) consisting of five developmentally stable DNaseI hypersensitive sites (HSs) of which HS1 to HS4 are erythroid-specific [63]. This regulatory element is known to bind multiple erythroid and ubiquitous transcription factors and to mediate a critical enhancer function required for developmentally regulated globin gene expression. A unique property of the LCR is its ability to confer integration position-independent expression on a linked gene, however only HS2 act as a classical enhancer element [3]. In addition to the LCR, we tested gene-specific regions including approximately $5000 \mathrm{bp}$ upstream of the $H B G 2$ capsite, to 5000 bp downstream of the $H B G 1$ gene (Figure 6A). These regions were investigated for binding sites of protein- 1 genes silenced $>1.5$-fold from day 7 to day 28 .

We identified 22 transcription factors with potential binding sites in the LCR and $\gamma$-globin gene regions. For example, the GATA factors (GATA1, 2 and 3) which are essential for normal hematopoiesis. GATA2 expression overlaps with GATA1 during erythroid, megakaryocytic, eosinophilic and mast cells development [64]. Consistent with our prediction model for profile-1 genes (Additional file 8: Table S7), GATA2 over expression in K562 cells mediates activation of the erythroid-specific genes $\alpha$ - and $\gamma$-globin [65]. In committed erythroid progenitors, GATA2 is replaced by GATA1. Both GATA factors co-exist in various protein complexes such as SCL/ LMO2/E2A/Ldb-1 and NFE2 which have been demonstrated to bind directly to globin gene promoters [66]. 


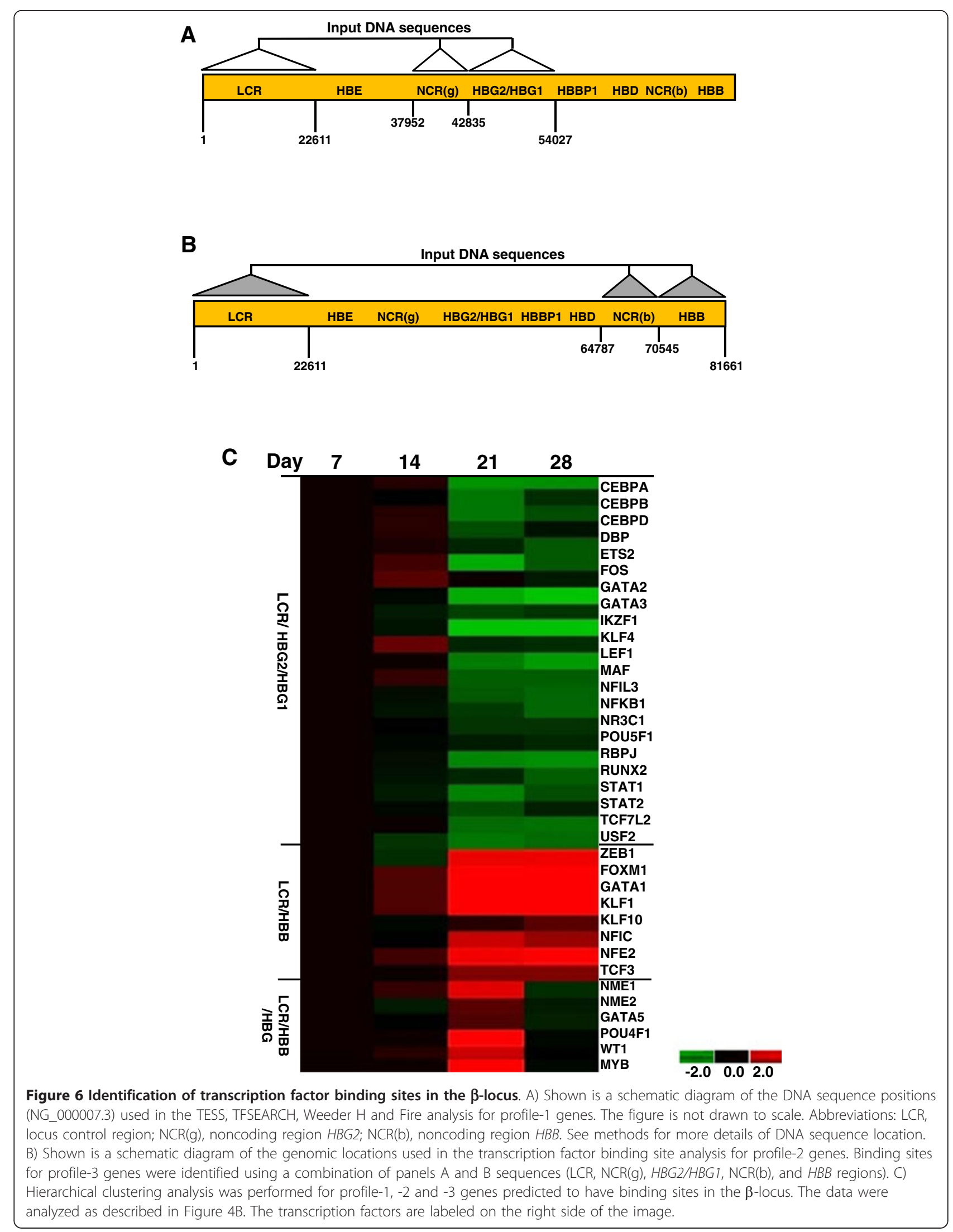


However, GATA3 is expressed mainly in T-cells [64] and a direct role in globin regulation has not been documented. Other factors such as $C / E B P A$, which binds as a homodimer to certain promoters, can also forms heterodimers with the related proteins $C / E B P B$ [67]. These proteins have been implicated as positive regulators of $\gamma$-globin through the distal CCAAT box.

In the DNA-binding site studies we also identified the signal transducers and activators of transcription (Stat) proteins. Stat1 and Stat 2 form heterodimers in response to erythropoietin stimulation, however, others have reported that Stat $1 \alpha$ and Stat 3 can act as negative regulators in erythropoietin-induced erythroid differentiation [68]. Our laboratory demonstrated a negative role for Stat $3 \beta$ in $\gamma$-globin expression while Stat $3 \alpha$ played a positive role [69]. Subsequent studies from our group demonstrated that Stat3 competes with GATA1 binding to an element located between +9 to +16 in the $\gamma$-globin gene 5' untranslated region [70].

The subsequent in silico analysis for profile- 2 genes was conducted with sequences in the LCR and about 5700 bp upstream of $H B B$ to the end of the $\beta$-locus (Figure 6B and Additional file 9: Table S8). Several factors known to be involved in hematopoiesis were identified such as the lineage-restricted transcription factors GATA1, SCL/Tal1, LMO2, LDB1 and KLF1 [71]. As expected, we identified $K L F 1$ the most extensively characterized regulator of $H B B$ expression and erythrocyte development [32]. It is a zinc finger protein that recognizes a subset of CACC motifs and acts primarily as a transcriptional activator [72]. Recent data demonstrated a role for KLF1 in BCL11A activation to produce $\gamma$-globin silencing [73]. Interestingly, our microarray data show a 20.6-fold increase in BCL11A expression from day 7 to day 28 (Additional file 6: Table S5) which is consistent with its demonstrated role as a $\gamma$-globin silencer [74-76]. In the $\beta$-locus, BCL11A is bound in HS3, approximately $3 \mathrm{~kb}$ downstream of $H B G 1$ and upstream of $H B D$ [74]. The specificity of the BCL11A binding site $5^{\prime} \mathrm{CCAC}(\mathrm{c} / \mathrm{g})$ is variable [6]. In our in silico analysis, BCL11A was not predicted to bind the LCR or downstream HBG1 regions. This may be due to variations in the consensus binding sites and/or differences in the software algorithms used in the four platforms to identify binding sites. Our design did not include the $H B D$ region and only transcription factors with predicted binding sites in all programs were retained on the list. Alternatively, BCL11A may not directly bind in the $\beta$ locus rather produce its effect by protein-protein interactions. This mechanism is supported by the ability of BCL11A to interact with GATA-1, HDAC1 and HDAC2 [74] and Sox 6 [77] among other proteins. On the other hand, we identified the known NFE2 binding sites in HS2; NFE2 recognizes the TCAT/C sequence of the AP-1-like core palindrome sequence present in a number of erythroid genes. NFE2 also has been shown to play an important role in $\beta$-globin gene regulation [78].

Finally, for profile-3 genes we analyzed all regions described for profile- 1 and -2 genes reasoning that factors associate with the $\gamma / \beta$ switch might bind throughout the $\beta$-locus. We identified six transcription factors (Additional file 10: Table S9) with predicted binding sites upstream of HS1 (NME1), in HS3 (NME2), both $\gamma$ globin genes (POU4F1), 5 ' of $\beta$-globin (MYB and GATA5) and in 5' HS3 (WT1). One of the most important regulators of mammalian hematopoiesis is c-MYB, an evolutionarily conserved transcription factor [79] highly expressed in immature hematopoietic cells and down-regulated during differentiation. We observed peak expression around day 21 consistent with published data. Bianchi and colleagues [80] demonstrated that c-MYB silencing in CD $34^{+}$stem cells increased commitment toward the macrophage and megakaryocyte lineages, whereas erythroid differentiation was impaired. Furthermore, gene expression profiling analysis identified KLF1 and LIM Domain Only 2 as putative targets, which could account for the effects of c-MYB knockdown. A genome-wide association study has shown polymorphisms in the HBS1L-MYB intergenic (HMIP) region are highly associated with elevated fetal hemoglobin levels in Chinese $\beta$-thalassemia heterozygotes [81]. The exact mechanism by which these variants result in elevated fetal hemoglobin remains unclear, although it has been suggested MYB may mediate this effect [82].

The Wilms' tumor protein Wt1 is another interesting profile- 3 gene with predicted binding in the $\beta$-locus. It is required for embryonic development and has been implicated in hematologic disorders [83]. Wt1 deficiency may also compromise the proliferation and differentiation of erythroid progenitor cells [84]. A cis-element in the erythropoietin receptor promoter of human and mouse genes was identified by mutation analysis. The authors conclude that activation of the erythropoietin receptor gene by Wt1 may represent an important mechanism in normal erythropoiesis.

Our findings suggest that transcription factors identified with potential binding sites in the $\beta$-locus by in silico analysis (Figure 6C) may have biological relevance to $H B G$ and $H B B$ gene regulation. We tested our hypothesis by analyzing two factors KLF4 (Figure 3B) and KLF12; in a recently published study [85]. By electrophoresis mobility shift assay, we demonstrated that KLF4 and KLF12 directly bind the $\gamma$-globin CACCC element. However, only KLF4 mediated positive regulation of $\gamma$-globin expression in cell lines and primary erythroid progenitors. Interestingly, the in silico studies only 
identified KLF4 binding in the $\gamma$-globin promoter (Additional file 8: Table S7).

Additional support for our experimental approach can be gained from $C / E B P$ proteins which have been demonstrated to activate $\gamma$-globin through binding of the distal CCAAT box [67]; C/EBP compete for binding of the repressor protein CCAAT displacement protein [86]. In fact, this is the proposed mechanism for hereditary persistence of fetal hemoglobin due to a C/T mutation at -117 in the distal CCAAT box [87]. These findings provide evidence that other transcription factors involved in globin gene regulation can be identified in our system.

\section{Conclusions}

The one-phase liquid culture system was used to define the erythroid transcriptome associated with the $\gamma / \beta$-globin gene switch in vitro and to address global mechanisms involved in globin gene regulation. We utilized primary erythroid cells to characterize three major gene expression patterns including profile- 1 genes silenced from day 7 to day 28, profile- 2 genes activation from day 7 to day 28 and profile- 3 genes with peak expression at day 21 around the time of the $\gamma / \beta$-globin switch. After erythroid differentiation the transcriptome was established using the Illumina BeadChip microarray platform. Profile-1 genes were related to hemopoeisis and the NF-K $\beta$, Interleukin-4 and p38 MAPK signaling pathways. Profile- 2 genes were shown to be associated with cell proliferation, heme synthesis and erythrocyte differentiation. Finally profile-3 genes were associated with nucleotide metabolism, and protein switching. The biological subgroups generated by our data corroborate published studies related to erythroid maturation and hemoglobin synthesis during terminal differentiation. New insights into the switching process will be obtained by further investigation of biological subgroups associate with the $\gamma / \beta$ switch observed at day 21 in culture.

\section{Methods}

\section{Primary erythroid culture system}

Peripheral blood mononuclear cells were isolated from three normal donors (Carter Blood Center, Bedford, TX) using Histapaque-1.077 (Cellgro Inc.). The mononuclear cells were grown in three independent cultures using the one-phase protocol as previously published [18]. Briefly, cells were cultured in $\alpha$ MEM containing $30 \%$ fetal bovine serum (Atlanta Biologicals, Atlanta GA), $1 \%$ deionized BSA with penicillin $(100 \mathrm{U} / \mathrm{mL})$ and streptomycin $(0.1 \mathrm{mg} / \mathrm{mL})$ at $37^{\circ} \mathrm{C}$ and $5 \% \mathrm{CO}_{2}$. The following growth factors were added on day 0: stem cell factor $(50 \mathrm{ng} / \mathrm{mL})$, Interleukin-3 $(10 \mathrm{ng} / \mathrm{mL})$ and erythropoietin $(4 \mathrm{U} / \mathrm{mL})$. See details of our one-phase culture protocol in Additional file 11: Supplemental
Methods. Approximately 1.5 million cells were harvested every 2-3 days from each culture for RNA isolation and morphological studies by cytospin preparations fixed in $100 \%$ methanol and stained with Giemsa. Cell counts and morphology were performed on a light microscope under oil emersion; at least 500 cells were counted on each slide.

\section{RT-qPCR analysis}

The mRNA levels of $\gamma$-globin, $\beta$-globin, and glyceraldehyde-3-phosphate dehydrogenase (GAPDH) were measured as previously published $[52,53,85]$ to establish the $\gamma / \beta$ globin switch in culture. Total RNA was extracted from $5 \times 10^{6}$ cells at each time point from the three independent cultures using a Trizol kit (Invitrogen, Carlsbad, CA) according to the manufacturer's instructions. RNA qualities were confirmed by Bio-Rad Experion automated electrophoresis. Based on published criteria all samples used in our analysis had RQI values > 8.0. cDNA was prepared from total RNA $(1 \mu \mathrm{g})$ using the Improm-II reverse transcriptase (RT) system (Promega, Madison, WI). The $\gamma$-globin, $\beta$-globin, and GAPDH mRNA levels were quantified by qPCR (iCycler iQ; Bio-Rad, Hercules, CA) and standard curves were generated using a Topo7-based plasmid carrying the $\gamma$ globin (Topo7- $\beta$-globin), Topo7- $\beta$-globin, or Topo7GAPD cDNA.

\section{Illumina BeadChip microarray analysis}

Total RNA isolated on day 7, 14, 21 and 28 was used for microarray analysis on the whole-genome Illumina HumanWG-6V2 Expression BeadChip with 48,700 probes (Illumina, Inc., San Diego, CA) per the manufacturer's protocol. Briefly, RNA samples $(0.5 \mu \mathrm{g})$ were amplified using Illumina TotalPrep RNA amplification kit (Enzo, Austin, TX) with biotin UTP labeling. Single stranded cDNA was generated using a T7 oligo(dT) primer followed by second strand synthesis to generate double-stranded cDNA which was column purified. Biotin-labeled cRNA was synthesized by in vitro transcription using T7 RNA polymerase which was column purified, and checked for quality using the Bio-Rad Experion system (Hercules, CA). cRNA $(1.5 \mu \mathrm{g})$ was hybridized to the Illumina BeadChip per protocol and streptavidin-Cy3 (Amersham, Piscataway, NJ) was used for detection. Chips were scanned on an Illumina Beadstation.

The following RT-qPCR approach confirmed the microarray data. Total RNA was isolated using the Trizol kit (Invitrogen) according to the manufacturer's instructions. RNA solutions were treated with DNase I before RT then cDNA was synthesized in a reaction containing SuperScript III RT (Invitrogen) and random hexamer primers. Gene specific primers were designed 
using Primer3 software http://frodo.wi.mit.edu/primer3/. PCR reactions were performed using a SYBR PCR master mix kit (AB Biosystems, Inc. Carlsbad, $C A$ ), and a Chromo4 Fluorescence Detector (Bio-Rad). The PCR protocol included denaturation at $95^{\circ} \mathrm{C}$ for $10 \mathrm{~min}$, followed by 40 cycles of $95^{\circ} \mathrm{C}$ for $15 \mathrm{~s}$ and $60^{\circ} \mathrm{C}$ for $1 \mathrm{~min}$ and $18 \mathrm{~S}$ or GAPDH RNA were used as internal controls. Serial dilutions of cDNAs generated from reference RNA (Strategene Inc., La Jolla, CA) were used to establish standard curves for each gene. The qPCR results were analyzed using Opticon software (Bio-Rad).

\section{Bioinformatics and biostatistics analysis}

The raw data obtained from the scanner were summarized as probe level signal intensities using Illumina BeadStudio v2.1.3 (Illumina), then background subtraction and quantile normalization (Additional file 1: Figure S4A) were completed using the MBCB algorithm [88]. After data normalization, we performed time-course analysis with PCA software (NIA Array Analysis Tool http://gsun.grc.nia.nih.gov/ANOVA/bin/login.cgi) to characterize three major genomic profiles [89]. For PCA, the genomics data were mined to follow the expression patterns of $\gamma$ - and $\beta$ - globin during erythropoiesis which is best analyzed based on the F-distribution with ANOVA $=0.01$ and FDR $=0.05$. Because we wanted to maintain globin gene profiles during our evaluation, permutation analysis was not conducted. The different profiles were analyzed at the $>1.25$-fold, $>1.5$ fold, and $>2.0$-fold change levels in a time-course manner on day $7,14,21$ and 28 to define three major gene profiles. For subsequent bioinformatics analyses, we used genes with $>1.5$-fold change in expression (Additional file 6: Table S5).

To support the results of PCA, Hierarchical Clustering methods were also used. Profile-1, profile- 2 and profile3 with 1.25-fold, 1.5-fold, and 2.0-fold change were input into BRB ArrayTools http://linus.nci.nih.gov/BRBArrayTools.html[90]. After log-transformation the intensity values were loaded into the Gene Cluster 3.0, BRB platform. The various genes (rows) and culture times (columns) were clustered using the correlation distances with similarity metric and average linkage. Finally, a heat-map was generated using the average gene expression fold-change values at the different time points to demonstrate up-regulated genes (red), gene silencing (green) and day 7 normalized to one or no change in gene expression (black). Three major genomic profiles including profile-1 (gene silencing from day 7-28), profile-2 (gene activation from day 7-28) and profile- 3 with peak expression at day 21 were generated. These data are available through the National Center for Biotechnology Information Gene Expression Omnibus [91] using accession number ID GSE35102.
To validate the microarray data we chose a subset of genes to analyze by RT-qPCR and performed statistical analysis to determine the correlation between microarray and RT-qPCR data. Subsets of profile-1 and profile2 genes were analyzed using the correlation coefficient, $\mathrm{R}^{2}$ and confidence intervals of parameter were also generated in the statistical analysis. Descriptive statistics for microarray and RT-qPCR data including raw counts, means and standard error were used to present the distribution of the measured parameters. Statistical assessment of these characteristics at different time points for microarray was performed using the Student's $t$-test ( $p$ $<0.05$ was considered significant).

\section{DAVID GO analysis}

DAVID GO software http://david.niaid.nih.gov was used to classify the large number of genes mined into biological functional groups. The gene subgroups were loaded into the GO platform and then a) the data were analyzed and Kappa scores calculated to evaluate the mining process; b) the transcriptome of each profile was categorized by biological process and molecular function using an over-representation analysis.

\section{Functional genomics analysis}

The pathway analyses from the mined genes were performed using IPA (IPA Ingenuity Systems, Inc., Redwood City, CA) software. Core analysis was processed using direct and indirect relationships for pathway scoring. Profile-1, profile-2, and profile-3 genes were investigated using the IPA software according to the manufacturer's instruction. The final data were reported by Pathway map and "txt" version with a hit list; Pvalues $<1.3 \log (-)$ was used as a cut off for statistical significance [91].

\section{Transcription factor analysis}

To search for putative transcription factor binding sites in the $\beta$-globin locus on chromosome 11 the following software programs were used: TESS (http://www.cbil. upenn.edu/cgi-bin/tess/tess[92], TFSEARCH http://mbs. cbrc.jp/research/db/TFSEARCH.html) [93], FIRE http:// quantbio-tools.princeton.edu/cgi-bin/FIRE/form.pl[94] and Weeder $\mathrm{H}$ version 1.0 [95]. The Genome Browser http://genome.ucsc.edu/ was employed to confirm motif coordinates in the Human Genome (version Hg 19). DNA sequences from the LCR, the 5' upstream $H B G 1$ region, coding sequence for $H B G 1$ and $H B G 2$, and 3' downstream sequence (Figure 6A) were analyzed with the four platforms to identify putative binding sites for profile-1 genes. A second analysis of the LCR, $H B B 5^{\prime}$ upstream, coding and 3' downstream regions was completed to identify putative binding sites for profile- 2 genes. Potential transcription factor binding sites for 
profile- 3 genes were analyzed in a combination of the regions tested for profile- 1 and -2 genes.

\section{Additional material}

Additional file 1: Figure S1. RNA quality check. Figure S4A Multiple regression analysis. Figure S5C Mitotic Roles of Polo-Like Kinasae signaling pathway. Figure S5D ATM signaling pathway.

Additional file 2: Table S1. Day 7 Illumina HumanWG-6V2 Expression BeadChip raw data. This file contains a table listing microarray raw data including the mean (M) and standard error (SEM) for each gene calculated from triplicate RNA samples extracted on day 7 from cells grown in the one-phase liquid culture system. Briefly, total RNA with amplified and biotin-labeled and then used for hybridization to the Illumina HumanWG-6V2 Expression BeadChip. Microarray raw data were generated on the Illumina Beadstation scanner.

Additional file 3: Table S2. Day 14 Illumina HumanWG-6V2 Expression BeadChip raw data. This file contains a table listing microarray raw data including the mean (M) and standard error (SE) for each gene calculated from triplicate RNA samples extracted on day 14 from cells cultured in the one-phase liquid culture system. Briefly, total RNA with amplified and biotin-labeled and then used for hybridization to the Illumina HumanWG-6V2 Expression BeadChip. Microarray raw data were generated on the Illumina Beadstation scanner.

Additional file 4: Table S3. Day 21 Illumina HumanWG-6V2 Expression BeadChip raw data. This file contains a table listing microarray raw data including the mean (M) and standard error (SE) for each gene calculated from triplicate RNA samples extracted on day 21 from cells cultured in the one-phase liquid culture system. Briefly, total RNA with amplified and biotin-labeled and then used for hybridization to the Illumina HumanWG-6V2 Expression BeadChip. Microarray raw data were generated on the Illumina Beadstation scanner.

Additional file 5: Table S4. Day 28 Illumina HumanWG-6V2 Expression BeadChip raw data. This file contains a table listing microarray raw data including the mean (M) and standard error (SE) for each gene calculated from triplicate RNA samples extracted on day 28 from cells cultured in the one-phase liquid culture system. Briefly, total RNA with amplified and biotin-labeled and then used for hybridization to the Illumina HumanWG-6V2 Expression BeadChip. Microarray raw data were generated on the Illumina Beadstation scanner.

Additional file 6: Table S5. Differential gene expression generated by PCA. This file contains three tables listing genes with > 1.5-fold change in expression during erythroid maturation in our one-phase liquid culture system. PCA data show the log change; a positive value indicates decreased gene expression from day 7 to day 28 in contrast to increased gene expression indicated by a negative log change. The correlation score indicates the level of agreement between the specific globin gene profile curve and PCA values.

Additional file 7: Table S6. Gene Ontology analysis from profile-1, -2 and -3 gene subsets. This file contains a complete list of the DAVID GO terms generated for the three gene groups tested; the count number indicates how many genes were identified in the specific pathway by microarray analysis. The $p$ values indicate the significance level for the specific pathways identified. The Benjamin score represents the adjusted multiple $p$ values to take into account all genes involved in the pathways identified.

Additional file 8: Table S7. TESS, TFSEARCH, Weeder $\mathrm{H}$ and Fire analysis for profile-1 genes.

Additional file 9: Table S8. TESS, TFSEARCH, Weeder $\mathrm{H}$ and Fire analysis for profile-2 genes.

Additional file 10: Table S9. TESS, TFSEARCH, Weeder $\mathrm{H}$ and Fire analysis for profile-3 genes.

Additional file 11: Supplemental Methods. Method for the one-phase tissue culture system.

\section{Abbreviations}

ATM: Ataxia telangiectasia mutated gene; C/EBPA: CCAAT/enhancer-binding protein alpha; DBP: D site of albumin promoter binding protein; (HSs): DNasel hypersensitive sites; ERK/MAPK: Extracellular signal-regulated kinase/ mitogen-activated protein kinase; FOXM1: Forkhead box M1; FOXQ1: Forkhead box Q1; GATA1: GATA binding protein 1; GO: Gene ontology; IPA: Ingenuity pathway analysis; KLF1: Kruppel-like factor 1; (LCR): Locus control region; MBCB: Model-Based Background Correction for BeadArrays; NFE2: Nuclear factor erythroid 2; P38 MAPK: P38 mitogen activated protein kinase; PCA: Principal component analysis; STAT1: Signal transducer and activator of transcription 1; TESS: Transcription elements search system; TFSEARCH: Transcription Factor Search.

\section{Acknowledgements}

We would like to thank Dr. Hongyan Xu in the Department of Biostatistics at the Georgia Health Sciences University for review of the statistical analysis. This work was supported by grant HL069234 to Dr. Betty S. Pace. The authors would like to acknowledge the assistance of the Genomics Shared Resource at the Harold C. Simmons Cancer Center, UT Southwestern Medical Center, which is supported in part by an NCl Cancer Center Support Grant, 1P30 CA142543-01. The inclusion of trade names or commercial products in this article was solely for the purpose of providing specific information and does not imply recommendation for their products.

\section{Author details}

${ }^{1}$ Department Pediatrics, Georgia Health Sciences University, 1120 15th St. CN-4112, Augusta, GA 30912, USA. 'Department of Radiation Oncology, University of Texas Southwestern Medical Center, 5323 Harry Hines Blvd, Dallas, TX 75390, USA. ${ }^{3}$ Department of Psychiatry, University of Texas Southwestern Medical Center, 5323 Harry Hines Blvd, Dallas, TX 75390, USA.

\section{Authors' contributions}

BP and MS conceived, designed and guided the study and edited the manuscript. WL performed experiments including cell culture and drug inductions, and RNA specimen preparation. BL performed the bioinformatics analysis and drafted the manuscript. LD carried out the microarray processing and RT-qPCR confirmation and assisted with bioinformatics analysis. All authors read and approved the final manuscript.

\section{Competing interests}

The authors declare that they have no competing interests.

Received: 7 September 2011 Accepted: 26 April 2012 Published: 26 April 2012

\section{References}

1. Groudine M, Kohwi-Shigematsu T, Gelinas R, Stamatoyannopoulos G, Papayannopoulou T: Human fetal to adult hemoglobin switching: changes in chromatin structure of the beta-globin gene locus. Proc Natl Acad Sci USA 1983, 80:7551-7555.

2. Giardina B, Messana I, Scatena R, Castagnola M: The multiple functions of hemoglobin. Crit Rev Biochem Mol Biol 1995, 30:165-96.

3. Bungert J, Tanimoto K, Patel S, Liu Q, Fear M, Engel JD: Hypersensitive Site 2 specifies a unique function within the human $\beta$-globin locus control region to stimulate globin gene transcription. Mol Cell Biol 1999, 19:3062-3072.

4. Higgs DR, Vickers MA, Wilkie AO, Pretorius IM, Jarman AP, Weatherall DJ: A review of the molecular genetics of human alpha-globin gene cluster. Blood 1989, 73:1081-104.

5. Pauling L, Itano HA, Singer SR: Sickle-cell anemia, a molecular disease. Science 1949, 110:543-548.

6. Jones MM, Steinhardt J: Evidence of the incorporation of normally nonaggregating hemoglobins into crystalline aggregates of deoxy hemoglobin S. J Biol Chem 1982, 257:1913-20.

7. Kinney TR, Helms RW, O'Branski EE, Ohene-Frempong K, Wang W, Daeschner C, Vichinsky E, Redding-Lallinger R, Gee B, Platt OS, Ware RE: Safety of hydroxyurea in children with sickle cell anemia: results of the HUG-KIDS study, a phase I/II trial. Pediatric Hydroxyurea Group. Blood 1999, 94:1550-1554.

8. Bianchi N, Zuccato C, Lampronti I, Borgatti M, Gambari : Fetal Hemoglobin Inducers from the Natural World: A Novel Approach for Identification of 
Drugs for the Treatment of $\beta$-Thalassemia and Sickle-Cell Anemia. eCAM Advance Access 2009, 6:141-151.

9. Pace BS, Zein S: Understanding mechanisms of gamma-globin gene regulation to develop strategies for pharmacological fetal hemoglobin induction. Dev Dyn 2006, 235:1727-37.

10. Sreenivasulu N, Sunkar R, Wobus U, Strickert M: Array platforms and bioinformatics tools for the analysis of plant transcriptome in response to abiotic stress. Methods Mol Biol 2010, 639:71-93.

11. Evster KM, Lindahl R: Molecular medicine: a primer for clinicians. Part XII: DNA microarrays and their application to clinical medicine. SDJ Med 2001, 54:57-61.

12. Addya S, Keller MA, Delgrosso K, Ponte CM, Vadigepalli R, Gonye GE, Surrey S: Erythroid-induced commitment of K562 cells results in clusters of differentially expressed genes enriched for specific transcription regulatory elements. Mol Cell Biol 2003, 23:5031-5042.

13. Keller MA, Addya S, Vadigepalli R, Banini B, Delgrosso K, Huang H, Surrey S: Transcriptional regulatory network analysis of developing human erythroid progenitors reveals patterns of coregulation and potential transcriptional regulators. Physiol Genomics 2006, 28:114-28.

14. Peller S, Tabach Y, Rotschild M, Garach-Joshua O, Cohen Y, Goldfinger N, Rotter V: Identification of gene networks associated with erythroid differentiation. Blood Cells Mol Dis 2009, 43:74-80.

15. Singleton BK, Burton NM, Green C, Brady RL, Anstee DJ: Mutations in EKLF/ KLF1 form the molecular basis of the rare blood group $\ln (\mathrm{Lu})$ phenotype. Blood 2008, 112:2081-8.

16. Sripichai O, Kiefer CM, Bhanu NV, Tanno T, Noh SJ, Goh SH, Russell JE, Rognerud CL, Ou CN, Oneal PA, Meier ER, Gantt NM, Byrnes C, Lee YT, Dean A, Miller JL: Cytokine-mediated increases in fetal hemoglobin are associated with globin gene histone modification and transcription factor reprogramming. Blood 2009, 114:2299-306.

17. Merryweather-Clarke AT, Atzberger A, Soneji S, Gray N, Clark K, Waugh C, McGowan SJ, Taylor S, Nandi AK, Wood WG, Roberts DJ, Higgs DR, Buckle VJ, Robson KJ: Global gene expression analysis of human erythroid progenitors. Blood 2011, 117:96-108.

18. Uddin S, Ah-Kang J, Ulaszek J, Mahmud D, Wickrema A: Differentiation stage-specific activation of p38 mitogen-activated protein kinase isoforms in primary human erythroid cells. Proc Natl Acad Sci USA 2004, 101:147-152

19. Maier-Redelsperger M, Noguchi $C T$, de Montalembert M, Rodger GP, Schechter AN, Gourbil A, Blanchard D, Jais JP, Ducroca R, Peltier JY: Variation in fetal hemoglobin parameters and predicted hemoglobin $\mathrm{S}$ polymerization in sickle cell children in the first two years of life: parisian prospective study on sickle cell disease. Blood 1994, 84:3182-8.

20. Schroeder WA, Huisman TH, Shelton JR, Shelton JB, Kleihauer EF, Dozy AM, Robberson B: Evidence for multiple structural genes for the gamma chain of human fetal hemoglobin. Proc Natl Acad Sci USA 1968, 60:537-44.

21. Bard H, Widness JA, Ziegler EE, Gagnon G, Peri KG: The proportions of ggamma- and agamma-globins in the fetal hemoglobin synthesized in preterm and term infants. Pediat Res 1995, 37:361-364.

22. Cantor $A B$, Orkin $S H$ : Transcriptional regulation of erythropoiesis: an affair involving multiple partners. Oncogene 2002, 21:3368-76.

23. Filippone C, Franssila R, Kumar A, Saikko L, Kovanen PE, SöderlundVenermo $M$, Hedman $K$ : Erythroid progenitor cells expanded from peripheral blood without mobilization or preselection: molecular characteristics and functional competence. PLoS One 2010, 5:e9496.

24. Ikonomi P, Noguchi $C T$, Miller $W$, Kassahun $H$, Hardison $R$, Schechter AN: Levels of GATA-1/GATA-2 transcription factors modulate expression of embryonic and fetal hemoglobins. Gene 2000, 261:277-87.

25. Kodeboyina S, Balamurugan P, Liu L, Pace BS: cJun modulates Ggammaglobin gene expression via an upstream cAMP response element. Blood Cells Mol Dis 2010, 44(1):7-15.

26. Zhang P, Basu P, Redmond LC, Morris PE, Rupon JW, Ginder GD, Lloyd JA: A functional screen for Krüppel-like factors that regulate the human gamma-globin gene through the CACCC promoter element. Blood Cells Mol Dis 2005, 35:227-35.

27. Case SS, Huber P, Lloyd JA: The gammaPE complex contains both SATB1 and HOXB2 and has positive and negative roles in human gammaglobin gene regulation. DNA Cell Biol 1999, 18:805-17.

28. Basu P, Sargent TG, Redmond LC, Aisenberg JC, Kransdorf EP, Wang SZ, Ginder GD, Lloyd JA: Evolutionary conservation of KLF transcription factors and functional conservation of human gamma-globin gene regulation in chicken. Genomics 2004, 84:311-9.

29. Zafarana G, Rottier R, Grosveld F, Philipsen S: Erythroid overexpression of C/EBPgamma in transgenic mice affects gamma-globin expression and fetal liver erythropoiesis. EMBO J 2000, 19:5856-63.

30. Lopez RA, Schoetz S, DeAngelis K, O'Neill D, Bank A: Multiple hematopoietic defects and delayed globin switching in Ikaros null mice. Proc Natl Acad Sci USA 2002, 99:602-7.

31. Yamauchi M, Kawai S, Kato T, Ooshima T, Amano A: Odd-skipped related 1 gene expression is regulated by Runx2 and Ikzf1 transcription factors. Gene 2008, 426:81-90.

32. Tallack MR, Whitington T, Yuen WS, Wainwright EN, Keys JR, Gardiner BB, Nourbakhsh E, Cloonan N, Grimmond SM, Bailey TL, Perkins AC: A global role for KLF1 in erythropoiesis revealed by ChIP-seq in primary erythroid cells. Genomics Res 2010, 20:1052-63.

33. Horak CE, Mahajan MC, Luscombe NM, Gerstein M, Weissman SM, Snyder M: GATA-1 binding sites mapped in the beta-globin locus by using mammalian chlp-chip analysis. Proc Natl Acad Sci USA 2002, 99:2914-9.

34. Zhou Z, Li X, Deng C, Ney PA, Huang S, Bungert J: USF and NF-E2 cooperate to regulate the recruitment and activity of RNA polymerase II in the beta-globin gene locus. J Biol Chem 2010, 285:15894-905.

35. Welch JJ, Watts JA, Vakoc CR, Yao Y, Wang H, Hardison RC, Blobel GA, Chodosh LA, Weiss MJ: Global regulation of erythroid gene expression by transcription factor GATA-1. Blood 2004, 104:3136-47.

36. Nair PN, Golden T, Melov S: Microarray workshop on aging: mechanism of ageing and development. Func Genom Aging 2003, 124:133-138.

37. Morey JS, Ryan JC, Van Dolah FM: Microarray validation: factors influencing correlation between oligonucleotide microarrays and realtime PCR. Biol Proced Online 2006, 8:175-93.

38. Brunner AM, Yakovlev IA, Strauss SH: Validating internal controls for quantitative plant gene expression studies. BMC Plant Biology 2004, 4(14):1-7.

39. da Huang W, Sherman BT, Lempicki RA: Systematic and integrative analysis of large gene lists using DAVID bioinformatics resources. Nat Protoc 2009, 4:44-57.

40. Hulsegge I, Kommadath A, Smits MA: Two different approaches for Gene Ontology analysis. BMC Proceedings 2009, 3(Suppl 4):S10

41. Dennis Glynn Jr, Sherman Brad T, Hosack Douglas A, Yang J, Michael W, Baseler H, Lane C, Lempicki Richard A: DAVID: Database for Annotation, Visualization, and Integrated Discovery. Gen Biol 2003, 4:P3.

42. Mu Y, Ding F, Cui P, Hu SN, Chen XH: Transcriptome and expression profiling analysis revealed changes of multiple signaling pathways involved in immunity in the large yellow croaker during Aeromonas hydrophila infection. BMC Genomics 2010, 11:506-520.

43. Forget MA, Desrosiers RR, Gingras D, Beliveau R: Phosphorylation states of Cdc42 and RhoA regulate their interactions with Rho GDP dissociation inhibitor and their extraction from biological membranes. Biochem J 2002, 361:243-254.

44. Sennikov SV, Eremina LV, Injelevskaya TV, Krysov SV, Silkov AN, Kozlov VA: Cytokine-synthesizing activity of erythroid cells. Russ J Immunol 2001, 6:193-202.

45. McHale CM, Zhang LP, Hubbard AE, Zhao X, Baccarelli A, Pesatori AC, Smith MT, Landi MT: Microarray analysis of gene expression in peripheral blood mononuclear cells from dioxin-exposed human subjects. Toxicology 2007, 229:101-113.

46. Kirchmeyer M, Koufany M, Sebillaud S, Netter P, Jouzeau JY, Bianchi A: Alltrans retinoic acid suppresses interleukin-6 expression in interleukin-1stimulated synovial fibroblasts by inhibition of ERK $1 / 2$ pathway independently of RAR activation. Arthritis Res Ther 2008, 10:141-145.

47. Martin-Blanco E: p38 MAPK signalling cascades: ancient roles and new functions. Bioessays 2000, 22:637-645.

48. Steinbrech DS, Mehrara BJ, Saadeh PB, Greenwald JA, Spector JA, Gittes GK, Longaker MT: VEGF expression in an osteoblast-like cell line is regulated by a hypoxia response mechanism. Am J Physiol Cell Physiol 2000, 278: C853-C860.

49. Tamura K, Sudo T, Senftleben U, Dadak AM, Johnson R, Karin M: Requirement for p38alpha in erythropoietin expression: a role for stress kinases in erythropoiesis. Cell 2000, 102:221-231. 
50. Nagata Y, Takahashi N, Davis RJ, Todokoro K: Activation of p38 MAP kinase and JNK but not ERK is required for erythropoietin-induced erythroid differentiation. Blood 1998, 92:1859-1869.

51. Dean JL, Brook M, Clark AR, Saklatvala J: p38 mitogen-activated protein kinase regulates cyclooxygenase- 2 mRNA stability and transcription in lipopolysaccharide-treated human monocytes. J Biol Chem 1999, 274:264-269.

52. Sangerman J, Lee MS, Yao X, Oteng E, Hsiao CH, Li W, Zein S, OforiAcquah SF, Pace BS: Mechanism for fetal hemoglobin induction by histone deacetylase inhibitors involves gamma-globin activation by CREB1 and ATF-2. Blood 2006, 108:3590-9.

53. Ramakrishnan $\mathrm{V}$, Pace $\mathrm{BS}$ : Regulation of $\mathrm{Y}$-globin gene expression involves signaling through the p38 MAPK/CREB1 pathway. Blood Cells Mol Dis 2011, 47:12-22.

54. Ishii S, Kurasawa Y, Wong J, Lee LYY: Histone deacetylase 3 localizes to the mitotic spindle and is required for kinetochore-microtubule attachment. Proc Natl Acad Sci USA 2008, 105:4179-4184.

55. Crusselle-Davis VJ, Vieira KF, Zhou Z, Anantharaman A, Bungert J: Antagonistic regulation of $\beta$-globin gene expression by helix-loop-helix proteins USF and TFII-I. Mol Cell Biol 2006, 26:6832-6843.

56. Mankidy R, Faller DV, Mabaera R, Lowrey CH, Boosalis MS, White GL, Castaneda SA, Perrine SP: Short-chain fatty acids induce gamma-globin gene expression by displacement of a HDAC3-NCoR repressor complex. Blood 2006, 108:3179-86.

57. Kalfa TA, Pushkaran S, Zhang XL, Johnson JF, Pan D, Daria D, Geiger $H$, Cancelas JA, Williams DA, Zheng Y: Rac1 and Rac2 GTPases are necessary for early erythropoietic expansion in the bone marrow but not in the spleen. Haematologica 2010, 95:27-35.

58. Lee JS: Functional link between DNA damage responses and transcriptional regulation by ATM in response to a histone deacetylase inhibitor TSA. Cancer Res Treat 2007, 39:116-124.

59. Berger E, Rome S, Vega N, Ciancia C, Vidal H: Transcriptome profiling in response to adiponectin in human cancer-derived cells. Physiol Genomics 2010, 42:61-70.

60. Zhang S, Yajima H, Huynh H, Zheng J, Callen E, Chen HT, Wong N, Bunting S, Lin YF, Li M, Lee KJ, Story M, Gapud E, Sleckman BP, Nussenzweig A, Zhang CC, Chen DJ, Chen BP: ongenital bone marrow failure in DNA-PKcs mutant mice associated with deficiencies in DNA repair. J Cell Biol 2011, 193:295-305.

61. Schoenfelder S, Sexton T, Chakalova L, Cope NF, Horton A, Andrews S, Kurukuti S, Mitchell JA, Umlauf D, Eskiw CH, Luo Y, Wei CL, Ruan YJ, Bieker JJ, Fraser P: Preferential associations between co-regulated genes reveal a transcriptional interactome in erythroid cells. Nat Genet 2010, 42:53-61.

62. Fibach E, Prus E: Differentiation of Human Erythroid Cells in Culture. Curr Prot Immunol 2005, Chapter 22(Unit 22F.7).

63. Palstra RJ, de Laat W, Grosveld F: Beta-globin regulation and long-range interactions. Adv Genet 2008, 61:107-42.

64. Buck I, Morceau F, Cristofanon S, Heintz C, Chateauvieux S, Reuter S, Dicato M, Diederich M: Tumor necrosis factor a inhibits erythroid differentiation in human erythropoietin-dependent cells involving p38 MAPK pathway, GATA-1 and FOG-1 downregulation and GATA-2 upregulation. Biochem Pharm 2008, 76:1229-1239.

65. Harigae H, Okitsu Y, Yokoyama H, Fujiwara T, Inomata M, Takahashi S, Minegishi N, Kaku M, Sasaki T: Induction of erythroid-specific genes by overexpression of GATA-2 in K562 cells. Int J Hematol 2006, 84:38-42.

66. Anguita E, Hughes J, Heyworth C, Blobel GA, Wood WG, Higgs DR: Globin gene activation during haemopoiesis is driven by protein complexes nucleated by GATA-1 and GATA-2. EMBO J 2004, 23:2841-52.

67. Stamatoyannopoulos G, Grosveld F: Hemoglobin switching. In The molecular basis of blood disease. Volume 3. Edited by: Stamatoyannopoulos G, Majerus PW, Perlmutter RM, Varmus H. Philadelphia: Saunders; 2001

68. Kirito K, Uchida M, Takatoku M, Nakajima K, Hirano T, Miura Y, Komatsu N: A novel function of Stat 1 and Stat3 proteins in erythropoietin-induced erythroid differentiation of a human leukemia cell line. Blood 1998, 92:462-71

69. Foley HA, Ofori-Acquah SF, Yoshimura A, Critz S, Baliga BS, Pace BS: Stat3 beta inhibits gamma-globin gene expression in erythroid cells. J Biol Chem 2002, 277:16211-9.
70. Yao X, Dzandu K, Shartava A, Kodeboyina L, Pace BS: Characterization of Stat3 in the gamma-globin promoter: the role of DNA protein interactions in gene silencing. Exp Hematol 2009, 37:889-900.

71. Hattangadi SM, Wong P, Zhang L, Flygare J, Lodish HF: From stem cell to red cell: regulation of erythropoiesis at multiple levels by multiple proteins, RNAs, and chromatin modifications. Blood 2011, 118:6258-68.

72. Miller IJ, Bieker JJ: A novel, erythroid cell-specific murine transcription factor that binds to the CACCC element and is related to the Krüppel family of nuclear proteins. Mol Cell Biol 1993, 13:2776-86.

73. Zhou D, Liu K, Sun CW, Pawlik KM, Townes TM: KLF1 regulates BCL11A expression and gamma- to beta-globin gene switching. Nat Genet 2010, 42:742-4.

74. Sankaran VG, Menne TF, Xu J, Akie TE, Lettre G, Van Handel B, Mikkola HK, Hirschhorn JN, Cantor AB, Orkin SH: Human fetal hemoglobin expression is regulated by the developmental stage-specific repressor BCL11A. Science 2008, 322:1839-42.

75. Sankaran VG, Xu J, Ragoczy T, Ippolito GC, Walkley CR, Maika SD, Fujiwara Y Ito M, Groudine M, Bender MA, Tucker PW, Orkin SH: Developmental and species-divergent globin switching are driven by BCL11A. Nature 2009, 460:1093-1097.

76. Sankaran VG, Xu J, Orkin SH: Transcriptional silencing of fetal hemoglobin by BCL11A. Ann N Y Acad Sci 2010, 1202:64-8

77. Xu J, Sankaran VG, Ni M, Menne TF, Puram RV, Kim W, Orkin SH: Transcriptional silencing of \{gamma\}-globin by BCL11A involves longrange interactions and cooperation with SOX6. Genes Dev 2010, 24:783-98

78. Forsberg EC, Downs KM, Bresnick EH: Direct interaction of NF-E2 with hypersensitive site 2 of the beta-globin locus control region in living cells. Blood 2000, 96:334-9.

79. Soza-Ried C, Hess I, Netuschil N, Schorpp M, Boehm T: Essential role of cmyb in definitive hematopoiesis is evolutionarily conserved. Proc Natl Acad Sci USA 2010, 107:17304-8.

80. Bianchi E, Zini R, Salati S, Tenedini E, Norfo R, Tagliafico E, Manfredini R, Ferrari S: c-Myb supports erythropoiesis through the transactivation of KLF1 and LMO2 expression. Blood 2010, 116:e99-110.

81. Farrell JJ, Sherva RM, Chen ZY, Luo HY, Chu BF, Ha SY, Li CK, Lee AC, Li RC, Li CK, Yuen HL, So JC, Ma ES, Chan LC, Chan V, Sebastiani P, Farrer LA, Baldwin CT, Steinberg MH, Chui DH: A 3-bp deletion in the HBS1L-MYB intergenic region on chromosome $6 \mathrm{q} 23$ is associated with $\mathrm{HbF}$ expression. Blood 2011, 117:4935-45.

82. Wahlberg $K$, Jiang J, Rooks $H$, et al: The HBS1L-MYB intergenic interval associated with elevated $\mathrm{HbF}$ levels shows characteristics of a distal regulatory region in erythroid cells. Blood 2009, 114:1254-1262.

83. Ariyaratana S, Loeb DM: The role of the Wilms tumour gene (WT1) in normal and malignant haematopoiesis. Expert Rev Mol Med 2007, 9:1-17.

84. Kirschner KM, Hagen P, Hussels CS, Ballmaier M, Scholz H, Dame C: The Wilms' tumor suppressor Wt1 activates transcription of the erythropoietin receptor in hematopoietic progenitor cells. FASEB J 2008 , 22:2690-701.

85. Kalra I, Alam M, Choudhary P, Pace BS: KLF4 Activates gamma-Globin Gene Expression in Primary Erythroid Cells. Brit J Haematol 2011, 154:248-59.

86. Barberis A, Superti-Furga G, Busslinger M: Mutually exclusive interaction of the CCAAT-binding factor and of a displacement protein with overlapping sequences of a histone gene promoter. Cell 1987, 50:347-359.

87. Fucharoen S, Shimizu K, Fukumaki Y: A novel C-T transition within the distal CCAAT motif of the G gamma-globin gene in the Japanese HPFH: implication of factor binding in elevated fetal globin expression. Nucleic Acids Res 1990, 18:5245-5253.

88. Allen JD, Chen M, Xie Y: Model-Based Background Correction (MBCB): R methods and GUI for Illumina Bead-array data. J Cancer Sci Ther 2009, 1:25-27.

89. Alexei ASharov, Dawood BDudekula, Minoru S H Ko: A web-based tool for principal component and significance analysis of microarray data. Bioinformatics Application Note 2005, 21:2548-2549.

90. Zhao Y, Simon R: BRB-ArrayTools Data Archive for human cancer gene expression: a unique and efficient data sharing resource. Cancer Inform 2008, 6:9-15. 
91. Edgar R, Domrachev M, Lash AE: Gene Expression Omnibus: NCBI gene expression and hybridization array data repository. Nucleic Acids Res 2002, 30:207-10.

92. Schug J: Using TESS to predict transcription factor binding sites in DNA sequence. Curr Protoc Bioinformatics 2008, Chapter 2(Unit 2.6).

93. Heinemeyer T, Wingender E, Reuter I, Hermjakob H, Kel A, Kel O, Ignatieva E, Ananko E, Podkolodnaya O, Kolpakov F, Podkolodny N, Kolchanov N: Databases on Transcriptional Regulation: TRANSFAC, TRRD, and COMPEL. Nucleic Acids Res 1998, 26:364-370.

94. Olivier $E$, Noam $S$, Saeed T: A universal framework for regulatory element discovery across all genomes and data-types. Mol Cell 2007, 28:337-350

95. Giulio P, Paolo M, Giancarlo M, Graziano P: Weeder Web: discovery of transcription factor binding sites in a set of sequences from coregulated genes. Nucleic Acids Res 2004, 32:W199-W203.

doi:10.1186/1471-2164-13-153

Cite this article as: Li et al.: Characterization of the transcriptome profiles related to globin gene switching during in vitro erythroid maturation. BMC Genomics 2012 13:153.

\section{Submit your next manuscript to BioMed Central} and take full advantage of:

- Convenient online submission

- Thorough peer review

- No space constraints or color figure charges

- Immediate publication on acceptance

- Inclusion in PubMed, CAS, Scopus and Google Scholar

- Research which is freely available for redistribution

Submit your manuscript at www.biomedcentral.com/submit 